\title{
From episodic avalanching to continuous flow in a granular drum
}

\author{
N. J. Balmforth ${ }^{1}$. J. N. McElwaine ${ }^{2}$ (I) \\ Received: 5 July 2017 / Published online: 13 July 2018 \\ (c) The Author(s) 2018
}

\begin{abstract}
Experiments are conducted to study the transition from episodic avalanching (slumping) to continuous flow (rolling) in drums half full of granular material. The width and radius of the drum is varied and different granular materials are used, ranging from glass spheres with different radii to irregularly shaped sand. Image processing is performed in real time to extract relatively long time series of the surface slope derived from a linear fit to the granular surface. For the drums with glass spheres, the transition mostly takes the form of a blend of the characteristics of episodic avalanching and continuous flow, that gradually switches from slumping to rolling as the rotation rate increases. For sand, a hysteretic transition can be observed in which one observes prolonged episodic avalanching or continuous flow at the same rotation rate, spanning a window of rotation speeds. For drums with the smallest spheres ( $1 \mathrm{~mm}$ diameter), the transition takes the form of noise-driven intermittent switching between clearly identifiable phases of episodic avalanching or continuous flow. This style of transition is also found for the sand in either the largest or smallest drum (by volume). We formulate dimensionless groupings of the experimental parameters to locate the transition and characterize the mean surface slope and its fluctuations. We extract statistics for episodic avalanching, including angle distributions for avalanche initiation and cessation, the correlations between successive collapses, mean avalanche profiles and durations, and characteristic frequencies and spectra.
\end{abstract}

Keywords Granular media · Rotating drum · Granular avalanche

\section{Introduction}

The flow of grains in a horizontally rotating drum is one of the simplest experiments to perform, yet exemplifies most of the key features that complicate the dynamics of a granular medium [4,27]: as the drum rotates, phases of solid-like behavior can co-exist with liquid-like or gas-like phases or be transformed into them. Even when the mean flow field is steady, particles can traverse yield surfaces to become entrained into flow or deposited into a solid bed. Finally, flow can abruptly halt or begin to furnish extensive intermittent motion. Thus, the rotating drum is perhaps the archetypal granular experiment. However, despite decades of study, most aspects of drum flow cannot be explained by any one

\section{J. N. McElwaine}

james.mcelwaine@durham.ac.uk

N. J. Balmforth

njb@math.ubc.ca

1 Department of Mathematics, University of British Columbia, Vancouver, BC V6T 1Z2, Canada

2 Department of Earth Sciences, University of Durham, Durham DH1 3LE, UK model. For example, continuum models based on empirical friction laws [13] or kinetic theory [18] chiefly apply to steady or rapid flow conditions and have had some success in applications to rotating drums (e.g. $[15,20,29,30])$. Nevertheless, the models do not describe all the dynamics when there are unsteady transitions from solid-like to fluid-like behavior. Worse, the literature on drum dynamics contains a number of overly simplistic or inaccurate theories and a variety of conflicting observations and interpretations, painting a poorly quantified picture of one of the more fundamental granular flow configurations. A first step to remedy this situation is to obtain reliable, reproducible and accurate experimental data, exploiting the continuous operation and image processing ability of modern cameras and computers to generate long stationary time series and high-quality statistics, which is one of our current goals.

For a roughened cylinder, for which the granular material is unable to slide freely over the container, the lowspeed flows are popularly classified as either "slumping" or "rolling" $[16,27]$. The former consists of intervals of solidbody rotation that are interrupted by episodic avalanches and arises at the lowest rotation rates. The continuous steady flow 
of the rolling state emerges at higher rotation speeds. For both slumping and rolling, the granular surface often remains relatively flat and is well characterized by a "dynamic friction angle"; there is only ever a shallow superficial flowing layer, bordered from the rigidly rotating grains below by a yield surface. At yet higher rotation rates, the surface profile becomes nonlinear, with a characteristic S-shape; eventually, rapid, gas-like flow emerges associated with significant centrifuging.

The transition between episodic avalanching and continuous flow has been documented to depend on particle diameter (relative to drum diameter) and shape, the aspect ratio and fill fraction of the drum, effective gravity and degree of cohesion $[3,8,16,24,25,36]$. Several studies have attempted to qualitatively describe the transition in terms of the matching of two distinct timescales. For example, it has been argued $[17,27]$ that the transition occurs when the typical duration of an avalanche matches the time taken for the same amount of material to rotate rigidly through a comparable angular change. This criterion has some empirical support, as do some other qualitative criteria $[8,23,32,34]$. However, none of these conditions emerge as the prediction of a dynamical theoretical model, nor do these studies address the precise form of the transition as a bifurcation in dynamical behavior. Indeed, experimental studies often report a "transitional regime," with mixed characteristics of both slumping and rolling, but offer no quantitative details (e.g. [3,7,16]).

By contrast, it has also been stated that the transition has a hysteretic form [32]: as one increases the drum rotation rate, episodic avalanching persists up to a threshold, before switching abruptly to continuous flow. If one then lowers the rotation rate, the continuous flow regime only becomes interrupted by episodic avalanching at a second, somewhat lower threshold. Both flow states are possible over the window of rotation rates sandwiched between the two thresholds. This description is rather different from a dynamical melange of slumping and rolling.

A third perspective suggests that the transition is noisedriven, and detailed experimental observations indicate a "bifurcation by intermittency" [12]. In this scenario, there are again two co-existing states, but noise disrupts episodic avalanching at the higher rotation rates and terminates continuous flow at low speeds. Over the window of the transition, the two states remain distinct and clearly identifiable, but stochastic fluctuations prompt intermittent switches between them. It is not clear whether the mix of behavior reported in other studies corresponds to this intermittent switching, or whether the system dynamics was rougher, with no clear division into recognizable prolonged states of episodic avalanching or continuous flow.

A complementary theoretical approach is based on modeling the avalanching granular medium as a fluid-like continuum accelerating under gravity but retarded by solid-like friction $[4,33]$. The crudest models describe the dynamics in terms of a single evolution equation for the surface angle $\theta(t)$, allowing for switches in flow in the manner of a granular stick-slip friction law [28]. When static friction is higher than dynamic friction at the initiation of flow, and if the latter then increases with flow rate (as in traditional Bagnold-type friction laws), the models predict two possible flow states at low rotation rates: a periodic stick-slip-type motion reproducing the episodic avalanching state, and a steady state representing continuous flow. As one increases the rotation rate, the periodic stick-slip solution eventually disappears in a deterministic bifurcation, with the system then converging to the rolling state. The critical rotation rate at which the periodic oscillations disappear offers a rationalization of the transition from slumping to rolling. Moreover, the presence of two co-existing states at lower rotation speeds implies pronounced hysteresis. However, the continuous flow state exists for arbitrarily low rotation rates and there is no transition from rolling to slumping. This can be remedied by destabilizing the steady state in another deterministic bifurcation at low rotation rates by forcing the friction to start decreasing with flow speed $[4,33]$. Alternatively, noise can be added to the model to account for fluctuations due to the finite-size, granular nature of the medium. The continuous flow state is then disrupted at lower rotation rate and episodic avalanching at higher rotation rates without passing through any deterministic bifurcations, and the system can progress from one with pronounced hysteresis to one having an intermittent transition by raising the noise level [12].

In the current paper, we provide an experimental exploration of slumping and rolling and the transition between the two, examining in detail the effect of drum and particle geometry. We summarize the details of the experiment in Sect. 2, and then describe our results for glass spheres (Sect. 3) and sand (Sect. 4). Appendices contain some further technical details of the experiments, and a collection of additional results that back up our conclusions or provide extra information, but which we omit to streamline the main body of the paper.

\section{The experiment}

\subsection{Apparatus}

Our experiments were conducted using drums made from two acrylic cylinders fitted with a transparent front plate and a paper-covered back wall. For each, the position of the back wall could be adjusted to vary the drum width $W$ (axial length), and the inner cylindrical surface was covered with (60 grit) sandpaper to reduce any slip of the granular materials. Most of the experiments were conducted in the larger of the cylinders, with a diameter $D=287 \mathrm{~mm}$, which was 
Table 1 Dimensions of the drums, their ranges of rotation rate, and the particle diameters and characteristic angles of the granular materials

\begin{tabular}{|c|c|c|c|c|}
\hline \multirow{3}{*}{$\begin{array}{l}\text { Drum diameter (inner), } D(\mathrm{~mm}) \\
\text { Drum widths, } W(\mathrm{~mm}) \\
\text { Rotation rates, } \Omega \mathrm{rad} \mathrm{s}^{-1}\end{array}$} & & \multicolumn{2}{|c|}{$287,190,152,100$} & \multirow{2}{*}{$\begin{array}{l}137 \\
17,31,56,86\end{array}$} \\
\hline & & \multicolumn{2}{|c|}{$17,31,56,110,205$} & \\
\hline & & \multicolumn{2}{|c|}{$0.004-1$} & $0.01-1$ \\
\hline Nominal particle diameter $(\mathrm{mm})$ & Range $(\mu \mathrm{m})$ & $\theta_{1}$ & $\left\langle\theta_{\text {start }}\right\rangle \pm \sigma_{\text {start }}$ & $\left\langle\theta_{\text {stop }}\right\rangle \pm \sigma_{\text {stop }}$ \\
\hline \multicolumn{5}{|l|}{ Glass spheres } \\
\hline 1 & $783-1132$ & 21.3 & $21.7 \pm 0.1$ & $20.9 \pm 0.1$ \\
\hline 1.5 & $994-1483$ & 24.7 & $25.4 \pm 0.3$ & $24.3 \pm 0.2$ \\
\hline 2 & $1800-2200$ & 25.4 & $26.1 \pm 0.3$ & $24.9 \pm 0.2$ \\
\hline 3 & $2800-3200$ & 26.5 & $27.6 \pm 0.4$ & $25.8 \pm 0.3$ \\
\hline 5 & $4800-5200$ & 28.0 & $29.8 \pm 0.7$ & $26.9 \pm 0.5$ \\
\hline 10 & $9800-10300$ & 30.5 & $33.7 \pm 1.4$ & $28.3 \pm 1.2$ \\
\hline 16 & $15600-16500$ & 33.4 & $37.7 \pm 2.0$ & $30.1 \pm 1.6$ \\
\hline \multicolumn{5}{|l|}{ Sand } \\
\hline 1 & $624-1335$ & 36 & $38.7 \pm 0.3$ & $34.8 \pm 0.4$ \\
\hline
\end{tabular}

The spheres with $d \leq 5 \mathrm{~mm}$ are produced by Potters Industries in a range of diameters and no further sieving was performed. The two larger spheres $(d=10$ and $16 \mathrm{~mm})$ were commercial glass marbles for which we measured the diameter of spheres in representative samples directly with a caliper. Diameters less than $2 \mathrm{~mm}$ were measured in a Beckman Coulter LS13320 Laser Diffraction Particle Size Analyzer, which precisely measures the distribution. The size range is given by $d_{10}-d_{90}$ (the 10 and $90 \%$ percentiles, weighted by volume) for $d<2 \mathrm{~mm}$ and $d>5 \mathrm{~mm}$; for $d=2-5 \mathrm{~mm}$, we list the manufacturer's quoted size range. The characteristic angles are estimated using the largest drum $(D=287 \mathrm{~mm})$ with a width of $W=110 \mathrm{~mm} ; \theta_{1}$ is the surface angle at the commencement of continuous flow (Sect. 3.4); $\left\langle\theta_{\text {start }}\right\rangle \pm \sigma_{\text {start }}$ and $\left\langle\theta_{\text {stop }}\right\rangle \pm \sigma_{\text {stop }}$ are the mean starting and stopping angles during episodic avalanching, plus or minus their standard deviations (Sect. 3.5) rotated relatively precisely at a prescribed rate using friction rollers driven by a computer-controlled motor (see Appendix A). The front plate was made from glass. Cylindrical inserts with centering spacers were fabricated so that the diameter could be changed whilst using the same driving apparatus and data acquisition system. The smaller cylinder, with a diameter of about $137 \mathrm{~mm}$, had an acrylic front face and was mounted co-axially on a shaft driven directly by a geareddown motor. This second cylinder was used for a smaller number of more detailed measurements of the avalanching granular surface. The dimensions of the drums are provided in Table 1.

To eliminate one of the experimental parameters, in all experiments the drum was half filled with the granular media (the "fill fraction" was 0.5). We used glass spheres for the most part, with the range of diameters listed in Table 1. We also used a poly-disperse sand with a mean diameter of about a millimeter. Table 1 reports some characteristic friction angles for these materials. It is conventional to determine such angles by building sandpiles or tilting a plane layer. However, the statistics for surface slopes are far better measured in the rotating drum. For example, in the episodic avalanching regime at low rotation speed, we can extract satisfying statistics for the angles at which avalanching begins $\left(\theta_{\text {start }}\right)$ and ends $\left(\theta_{\text {stop }}\right)$. These quantities are random-looking variables with well-defined distributions; our measurements determine the mean values far more precisely than a sandpile experiment. One awkward issue is that the angles depend on drum width $W$ and diameter $D$ in our experiments (Sects. 3.4, 3.5); Table 1 quotes results for a relatively large drum with $D=287 \mathrm{~mm}$ and $W=110 \mathrm{~mm}$, for which at least the effect of the side walls is minimized. Mean angles of this type are also sensitive to ambient noise, dust and humidity. Indeed, a disturbing feature documented presently (Sect. 2.3) is a persistent ageing effect that leads to secular drifts of these angles as the drums rotate over long periods. Overall, despite their simple appeal, mean angle measurements of this sort are not robust measures of granular dynamics. We did not attempt to calibrate an empirical friction law like " $\mu(I)$ " using sheet flow down an inclined plane [14] (see also Appendix B).

The experiments were conducted in air-conditioned laboratories; the humidity was monitored to be $50 \pm 10 \%$ over their duration. Although the humidity was not precisely controlled, once the granular materials were loaded inside the drums, the arrangement was well sealed and so humidity was unlikely to vary during each suite of experiments (i.e. the updown sweeps described in Sect. 2.3). Nevertheless, humidity effects may have influenced the dynamics of some of smaller particles we used ( $1 \mathrm{~mm}$ glass spheres and the sand). The big drum was fixed to an optical table set on a workbench and included vibration shielding. The smaller drum was mounted on a heavy wooden board. No further effort was made to reduce ambient noise, with other devices in the laboratory in operation during the experiments. 


\subsection{Data analysis}

For each rotation rate, we used a machine vision camera, directly connected to a computer, to observe images of the granular medium through the front face of the drum. With a sampling rate of twenty or fewer frames per second, the images could be processed in real time, thereby avoiding the post-processing of excessive amounts of stored data. In particular, we used the contrast between the relatively bright particles and the back (matt black paper covered) wall to extract the location of the granular surface (defined by the upper edges of the top layer of grains) near the front glass face over a central section of the drum spanning about nine tenths of the total surface. The time series of the dynamical friction angle, $\theta(t)$, was then recorded exploiting a linear fit to the surface profile. Further details of the fitting process are summarized in Appendix A. For the $137 \mathrm{~mm}$ drum, we recorded movies of shorter duration and extracted the surface profile as a function of space and time.

In addition to the mean surface angle $\langle\theta\rangle$ (angular brackets denoting the time average), we also examine a number of other statistical measures extracted from the time series of $\theta(t)$, including the standard deviation, $\sigma$, and the distance skewness of its rate of change, $D_{\text {skew }}$ (see Appendix A). The standard deviation highlights the strength of slumping, but also measures the unsteadiness of the flow during rolling. The distance skewness detects chiefly episodic avalanching: in this regime the time series of $\theta(t)$ contains rising portions at fixed rate punctuated by falling sections with variable rate, which translates to a highly skewed signal that registers strongly in the $D_{\text {skew }}$ measurement.

For episodic avalanching, we record the starting and stopping angles of each collapse, $\theta_{\text {start }}$ and $\theta_{\text {stop }}$, and the avalanche "amplitude" $\Delta \theta=\theta_{\text {start }}-\theta_{\text {stop }}$. Practically, the angles are determined by detecting all the local extrema in the time series that are separated in time and amplitude by preset thresholds. The thresholding artificially deletes some of the smaller avalanches from the record; we chose the thresholds to be as small as possible to avoid such deletions but avoid the false detection of events due to noise in the signal (cf. Appendix A). The procedure records data during the rolling regime, which do not correspond to "starting" and "stopping" angles. These measurements can still be useful as they provide another diagnostic of the fluctuations during continuous flow. In fact, for all our experiments, $\langle\Delta \theta\rangle$ was closely related to $\sigma$.

\subsection{Burn-in}

To explore the transition from episodic avalanching to continuous flow, we performed sequences of experiments corresponding to sweeps of rotation rate $\Omega$, holding fixed the other experimental parameters. For each sweep, $\Omega$ was incrementally raised and then lowered, waiting at each rotation rate for well over a hundred episodic avalanches or particle circulations during continuous flow. At all but the very lowest speeds the drum made at least one complete rotation. Short sections at the beginning of each time series were deleted to remove transient adjustments occurring after the rotation rate was stepped up or down.

At the outset of our exploration, a pronounced ageing effect became evident that made the sweeps problematic to conduct. The ageing corresponded to a gradual systematic drift in the mean surface angle, $\langle\theta\rangle$, that we attribute to a combination of accrued damage to the surfaces of the particles and the associated generation of fine dust (secular drifts of surface slope have also been observed to occur due to the cohesive action of humidity, but typically for smaller particles than those used here [1]). For the bigger particles, the surface damage and dust were visible to the eye (for $d=16 \mathrm{~mm}$, the damage was substantial, leading us to limit our use of this material); for the smaller particles, the damage was much less noticeable, even under a microscope. Sample secular changes during repeated up-down sweeps with $d=3 \mathrm{~mm}$ glass spheres are shown in Fig. 1. Progressing from sweep to sweep, the mean angles drift to higher values; the net change is dramatic, exceeding a degree.

For the larger glass spheres and sand, even though the mean angle drifted during the repeated sweeps, the other statistical measures of the signal, $\sigma,\langle\Delta \theta\rangle=\left\langle\theta_{\text {start }}-\theta_{\text {stop }}\right\rangle$ and $D_{\text {skew }}$, showed little or no such trend; see Fig. $1 \mathrm{~b}-\mathrm{d}$. Thus, despite the drift, other key features of the drum dynamics were robust, including the rotation rates characterizing the transition between slumping and rolling. The transition, occurring for rotation rates near $\Omega \approx 2 \times 10^{-4} \mathrm{rad} \mathrm{s}^{-1}$ in the figure, is detected by the sharp change the diagnostics plotted in panels b-d. By contrast, for the smaller spheres with $d \leq 1.5 \mathrm{~mm}$, the mean angle drifted to smaller values as the material aged and all the statistical measures also changed, with the rotation rates marking the transition shifting to lower values as the sweeps progressed. Most disconcertingly, after two up-down sweeps, the transition had migrated to such low rotation rate that episodic avalanching could barely be observed (see Fig. 30 in the Appendix; hereon, we cite such figures using the superscript notation "( 30 ").

The drift of the mean angle could also be observed if the drum was rotated steadily at relatively high speed $(0.4 \mathrm{rad} / \mathrm{s})$. In such experiments, the drift largely subsided after a characteristic "burn-in" time for all the particles ${ }^{\circledR} 31$. This feature provided a convenient protocol for maturing particles to remove the problematic drift: starting with a fresh sample of particles, we first rotated the drum at constant, relatively high speed ( $\Omega=0.4 \mathrm{rad} \mathrm{s}^{-1}$ ), monitoring the mean angle. Once any drift had subsided (which usually required many hours), we terminated this preliminary "burn-in" experiment and then conducted the up-down sweep in rotation rate. This 


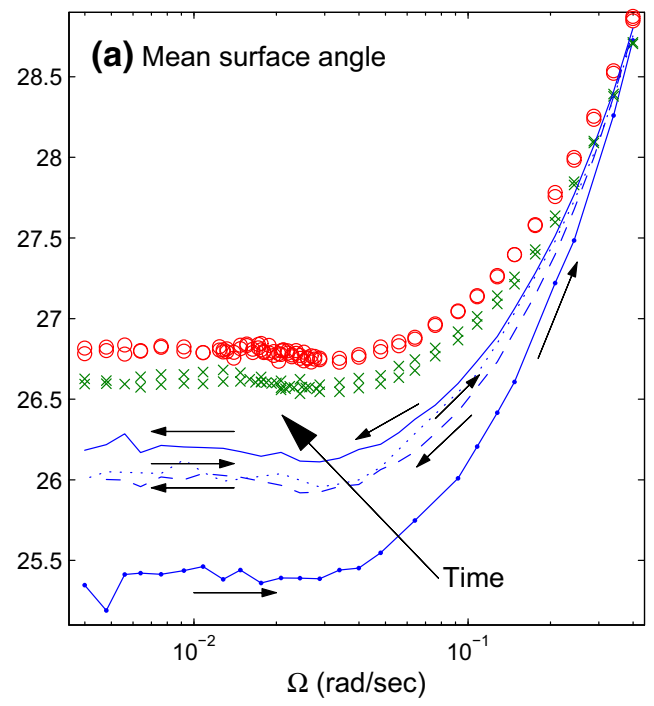

Fig. 1 Repeated sweeps with a fresh sample of $3 \mathrm{~mm}$ spheres in a drum with $(D, W)=(287,110) \mathrm{mm}$, showing a the secular increase of the mean surface angle $\langle\theta\rangle$, $\mathbf{b}$ the standard deviation $\sigma$ of the surface angle time series, $\mathbf{c}$ the mean avalanche amplitude $\langle\Delta \theta\rangle=\left\langle\theta_{\text {start }}-\theta_{\text {stop }}\right\rangle$, and (d) $D_{\text {skew }}$, the optimised distance skewness of $\dot{\theta}(t)$. For the sweeps, the

ensured that there was minimal secular change between the rising and falling parts of the sweep, enabling us to look unambiguously for any other forms of hysteresis. Results from two sweeps with different batches of matured $3 \mathrm{~mm}$ spheres are included in Fig. 1; the elimination of the secular drift leaves discrepancies of order 0.1 degrees between the two batches that we attribute to basic experimental error (slight differences in the fill fraction of the drum, the fit of the surface slope, camera or drum positioning and so forth).

The pervasive effect on the surface angle associated with surface damage does not appear to have been reported in detail previously in the literature on rotating drums, although it is clearly connected to the industrial process of polishing. As our main effort was to explore the transition from episodic avalanching to continuous flow, we ignored the phenomenon once eliminated by the burn-in. However, although the protocol successfully yielded repeatable, consistent results for the larger spheres with $d>1.5 \mathrm{~mm}$ and the sand, problems still remained for the smaller spheres. For $d=1.5 \mathrm{~mm}$ spheres it turned out that one could remove the particles from the drum after the first burn, wash and dry them, and then remature them in a second burn-in, ${ }^{(531}$ to obtain reproducible sweeps. For $d \leq 1 \mathrm{~mm}$, a similar maturation process still did not furnish adequately repeatable sweeps ${ }^{\circledR 30}$, leading us to report results chiefly for spheres with $d \geq 1.5 \mathrm{~mm}$.

To avoid repeating excessively long burn-in runs, we also recycled matured particles. In particular, we first matured particles in the drum with $(D, W)=(287,110) \mathrm{mm}$, and then used the matured batches for all the subsequent sweeps

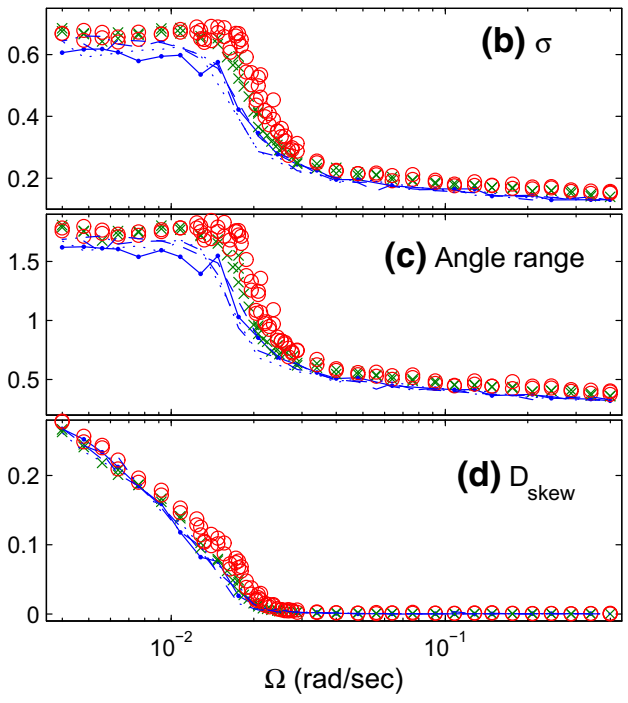

rotation rate is first stepped up (dots/solid line) and then stepped back down (dashed line); the up-down sequence is then repeated (dotted and solid lines). The circles and crosses show up-down sweeps for spheres aged according to our burn-in protocol

in drums with different width and radius. The recycled material still required some degree of burn-in (due perhaps to the loss of dust during removal and refilling of the drum), but typically less than half the time. The sandpaper lining the drums was also worn down by conducting repeated sweeps; we replaced the lining whenever wear became noticeable. No appreciable wear of the glass face of the big drum was discernible (the smaller drum was not used for sufficient time to wear the acrylic face.)

\section{The results for glass spheres}

\subsection{Phenomenology}

We first discuss the basic avalanche dynamics of the glass spheres. Figure 2 displays sample time traces of the surface angle $\theta(t)$ for $d=2 \mathrm{~mm}$ and varying rotation rate in the drum with $(D, W)=(287,31) \mathrm{mm}$. At the larger rotations rates, the motion takes the form of unsteady continuous flow with angle fluctuations of order a tenth of a degree. As $\Omega$ decreases, sporadic larger-amplitude fluctuations arise, which are the signature of incipient episodic avalanches. At first, these fluctuations correspond to unsteady flow, with material never coming to rest. Lowering the rotation speed further, however, leads to the momentary arrest of flow and the emergence of genuine episodic avalanches. These events become more frequent and well developed as one lowers $\Omega$ still further, until they dominate the time series. At no stage is 
Fig. 2 Sample time series of surface angle $\theta(t)$ for $2 \mathrm{~mm}$ spheres in the larger $(D=287 \mathrm{~mm})$ drum, with $W=31 \mathrm{~mm}$. Six time traces are shown (vertically offset), for the rotation rates indicated

Fig. 3 The intermittent transition for $1 \mathrm{~mm}$ spheres in the drum with $(D, W)=(289,110) \mathrm{mm}$
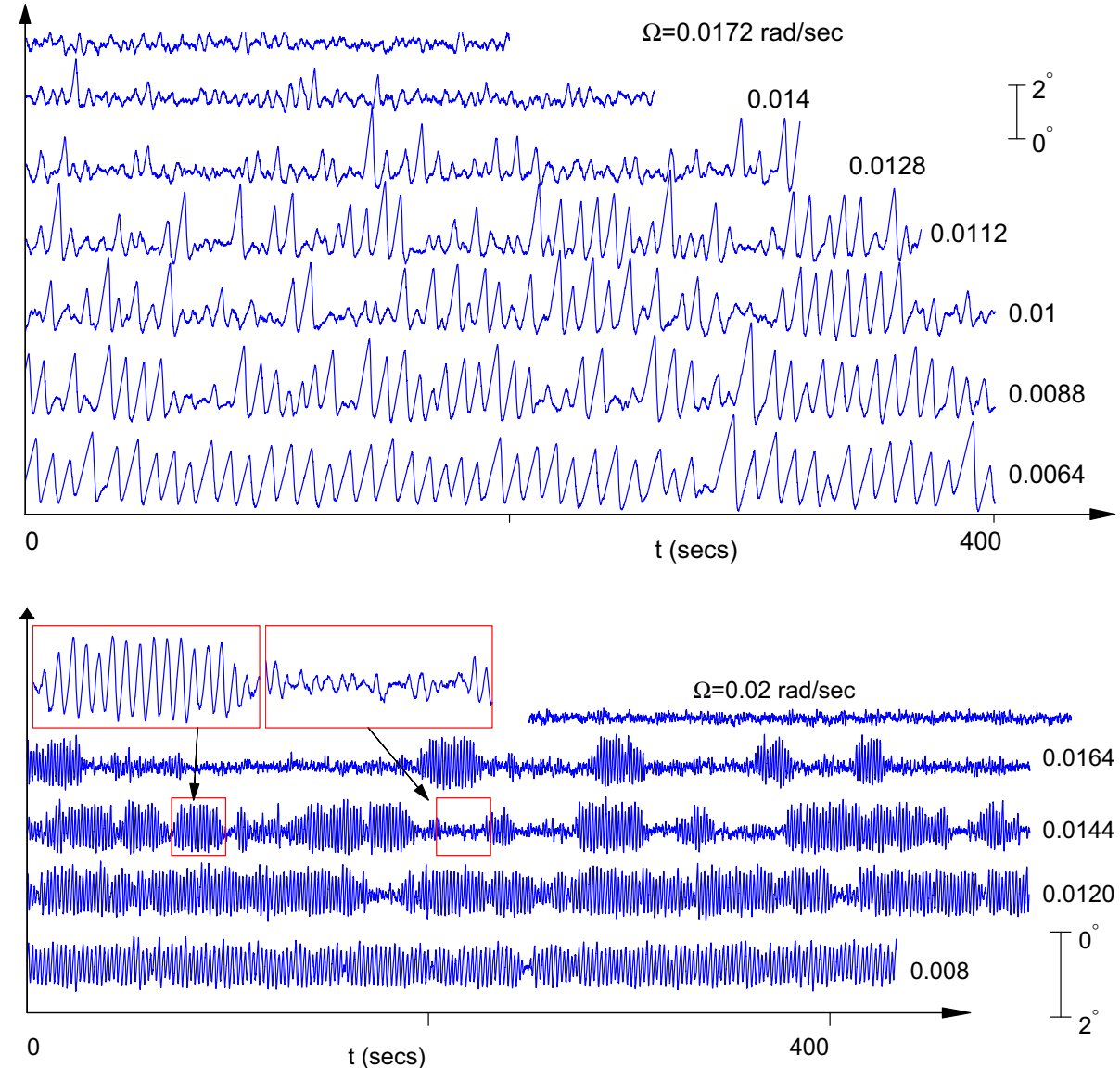

there an obvious alternation between prolonged, clear phases of either continuous flow or episodic avalanching. Hence there is a gradual transition from continuous flow to episodic avalanching, with no sign of any hysteresis if the rotation rate is varied up or down. This dynamics characterized all the spheres we used for the sweeps ( $d \geq 1.5 \mathrm{~mm}$ ); the melange of behavior is likely responsible for the loose descriptions of the transition appearing in existing literature.

The transition for the smallest particles $(d=1 \mathrm{~mm})$ took a clearer form, with intermittent switching between clearly identifiable periods of episodic avalanching and continuous flow; see Fig. 3. As the rotation rate increased through the transition, the typical residence time in episodic avalanching gradually dwindled until that state was replaced by uninterrupted continuous flow. Thus, for $d=1 \mathrm{~mm}$ the transition has a clear intermittent character [12], with the residence time in the two phases providing a diagnostic of the transition. For the rest of the spheres, the dynamics more effectively blended the features of the two states, rendering a residence time diagnostic difficult to extract.

Figure 4 displays sample space-time plots of the surface position for $d=2 \mathrm{~mm}$ spheres during both continuous flow and episodic avalanching. The time-averaged surface angle is subtracted off to emphasize the variations about that mean.
For the continuous flow (panel a), some residual coherent variations are still evident that are of low level and matched with fluctuations in the instantaneous surface slope (panel c). For the episodic avalanches (panel b), the space-time plot reveals the surface collapses and the intervals of rigid-body rotation. From such plots it is difficult to isolate any particular position at which an avalanche usually begins or ends: in some cases, the avalanche starts at one end of the surface; on others, collapse occurs first at drum center, or entire sections of the surface appear to mobilize simultaneously. Data collected from many avalanches suggested a weak bias of the starting position towards the top corner of the surface and the stopping position to the lower corner ${ }^{\circledR 32}$.

\subsection{Sweeps}

Up-down sweeps of the glass spheres for different particle radius and drum geometry are shown in Fig. 5; the gradual transition between episodic avalanching and continuous flow leads to a smooth connection between $\Omega$-independent statistics for low rotations rates to more systematic variations at higher speeds. Figure 5a shows data for the drum with $(D, W)=(287,110) \mathrm{mm}$, and varying particle diameter $d$. Fig. $5 \mathrm{~b}$, c show data for $3 \mathrm{~mm}$ spheres in drums with 


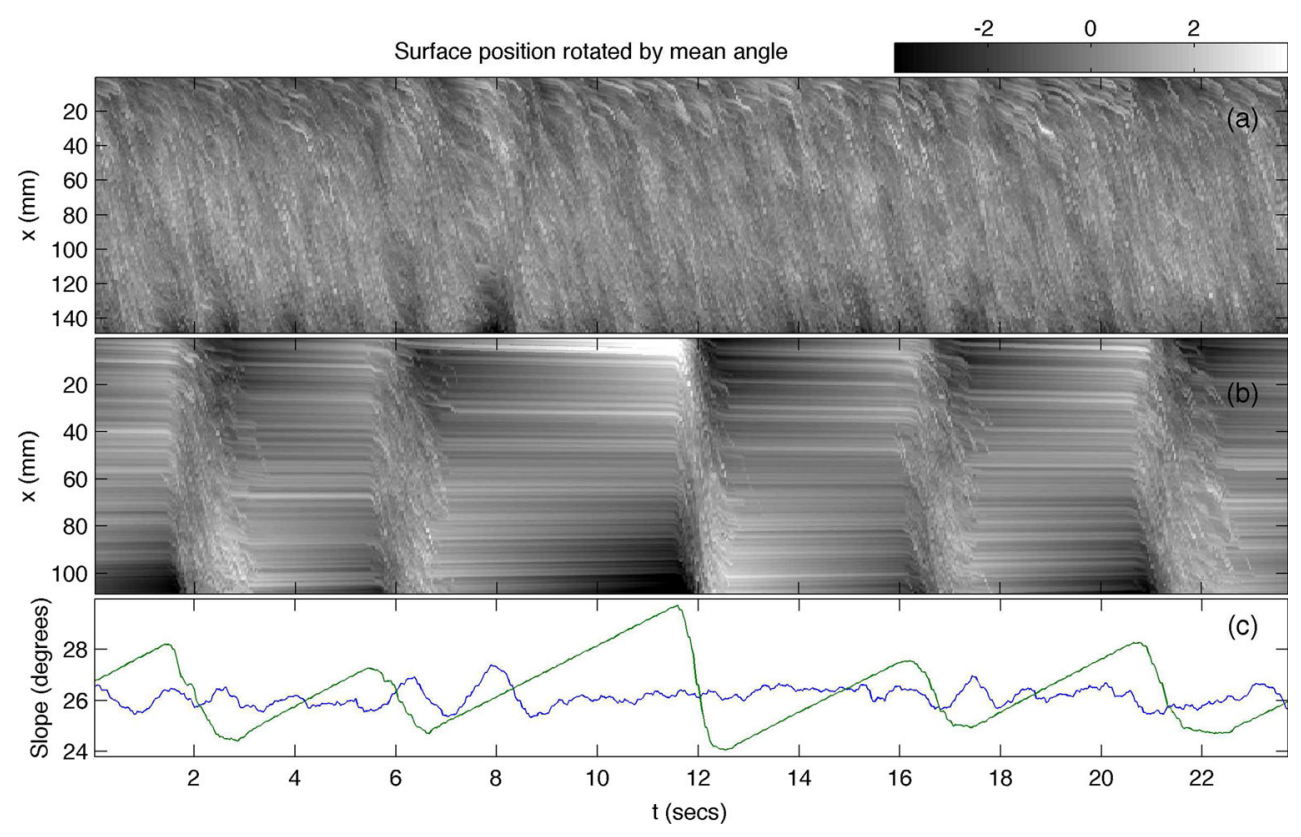

Fig. 4 Two experiments in the smaller drum $(D=137 \mathrm{~mm}, W=$ $31 \mathrm{~mm}, d=2 \mathrm{~mm}$ ) for $\mathbf{a} \Omega=0.079 \mathrm{rad} \mathrm{s}^{-1}$ and $\mathbf{b} \Omega=0.019 \mathrm{rad} \mathrm{s}^{-1}$. a, b Surface position (in $\mathrm{mm}$ ) as a density on the space-time plane, after rotation by the mean angle (with the position on the $y$-axis beginning at the top of the granular surface and lighter shading indicating higher relative elevations of the surface). $\mathbf{c}$ show the corresponding time series of surface angle $\theta(t)$ different diameter $D$ and width $W$, respectively. The figures illustrate how the rotation rates for transition vary significantly with $D$ and $d$, but not with $W$. Both the mean angle and its variability depend significantly on all of $D, W$ and $d$. In this figure, and in subsequent figures, each data point is an average over all experiments with the same parameters $(\Omega, d, D, W)$.

As illustrated in Fig. 5c, the mean angles and standard deviations become independent of $W$ once the drum is sufficiently wide. Such width-independent behavior appears roughly for $W>\frac{1}{3} D$, independently of particle diameter and shape (being similar for all the glass spheres and sand), and in agreement with previously presented data [5]. Many of the experimental drums used in previous studies are relatively narrow according to this criterion, with mean angles that are controlled by the side walls $[3,10,30]$. Despite this, the apparent independence of the flowing layer depth on $W$ as observed through the side wall is sometimes taken as evidence for width-independent dynamics. Although we did not directly measure this depth, it was apparent from our image statistics (specifically, the mean difference between consecutive images, which highlights flowing rather than rigidly rotating particles) that the flowing layer also did not vary significantly with $W$ at the front face of our drums. The situation is presumably similar to heap flows in a slot, for which it is argued that sidewall friction always controls the flowing layer depth and increases with $W$ until the slot width becomes comparable to the length of the apparatus [19]. Thus, obser- vations through the sidewall must be biased, with the flowing layer being much deeper than can be seen, as has been verified in NMR experiments [26].

Measuring mean angles through the front face of the drum can also be problematic, as surface slopes vary with axial position. For glass spheres, it has been reported that the surface angle changes by as much as $4^{\circ}$, with material piled up higher against the front and back walls [9]. By itself, this variation is not sufficient to explain the differences in mean angle for different $W$ observed for our drums (Fig. 5c). The characteristic range of the boundary effect is reported to be of the order of $0.14 D$, provided the particles are not too large. This suggests that the front and back wall are effectively isolated from one another when $W>0.28 D$, in agreement with our rough criterion $W>\frac{1}{3} D$. However, we made no attempt to quantify the axial variation of the surface slope in our drums.

\subsection{Locating the transition}

To locate the slumping-to-rolling transition, we use the diagnostics, $D_{\text {skew }}$ and $\sigma$. These quantities are collected together for all the sweeps with glass spheres in Figs. 6 and 7.

The $D_{\text {skew }}$ values in Fig. 6 can be used to locate the upper edge of the transition: we set $D_{\text {skew }}<D_{\text {crit }}$, with $D_{\text {crit }}=O\left(10^{-2}\right)$, to indicate uninterrupted continuous flow. The upper edge of the transition, $D_{\text {skew }}=D_{\text {crit }}$, then identifies a critical rotation rate $\Omega_{c}$ for each drum geometry and particle diameter. As shown by Fig. 6a, with $D_{\text {crit }}=0.02$, 


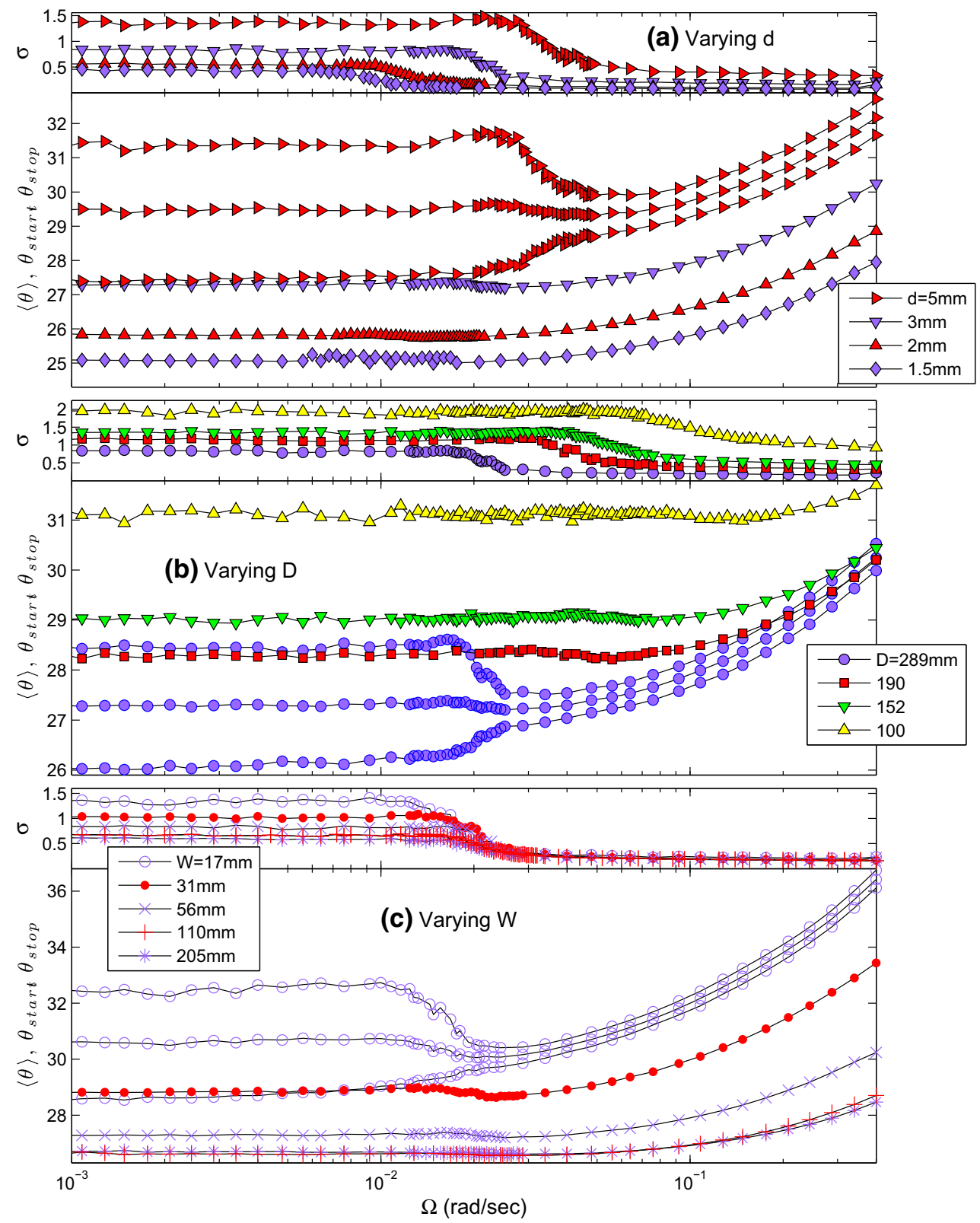

Fig. 5 Mean surface angles $\langle\theta\rangle$ and standard deviation $\sigma$, against rotation rate $\Omega$ for glass spheres. a Sweeps with the particle diameters indicated in the drum with diameter $D=289 \mathrm{~mm}$ and width $W=56 \mathrm{~mm}$. b Sweeps for $d=3 \mathrm{~mm}$ in drums with $W=56 \mathrm{~mm}$ and

the critical rotation rates $\Omega_{c}$ spread over an order of magnitude and vary significantly with the ratio $d / D$ of particle to drum diameter, but only very slightly with drum width. As shown in Fig. 6b, the spread in $\Omega_{c}$ can be largely suppressed by formulating the scaled Froude number,

$\mathrm{Fr}^{*}=\frac{\mathrm{Fr}}{1+\beta d / W}\left(\frac{D}{d}\right)^{\alpha}, \quad \mathrm{Fr}=\Omega \sqrt{\frac{D}{g}}$, the diameters $D$ indicated. c Sweeps for $d=3 \mathrm{~mm}$ in the drums with $D=287 \mathrm{~mm}$ and the widths $W$ indicated. In each panel, the mean starting and stopping angles for one of the sweeps is also shown, and each data point is averaged over all experiments with the same $(\Omega, d, D, W)$

with $\alpha \approx 1.1$ and $\beta=1.5$. The removal of drum-width dependence using the combination $\beta d / W$ is similar to previous adjustments of surface slope measurements (e.g. $[6,14]$ ), and is a device we exploit again below. With that factor in hand, the choice of $\alpha$ was then determined by a least squares fit. However, neither the inclusion of the factor $(1+1.5 d / W)^{-1}$ nor the difference of $\alpha$ from unity are especially significant. 


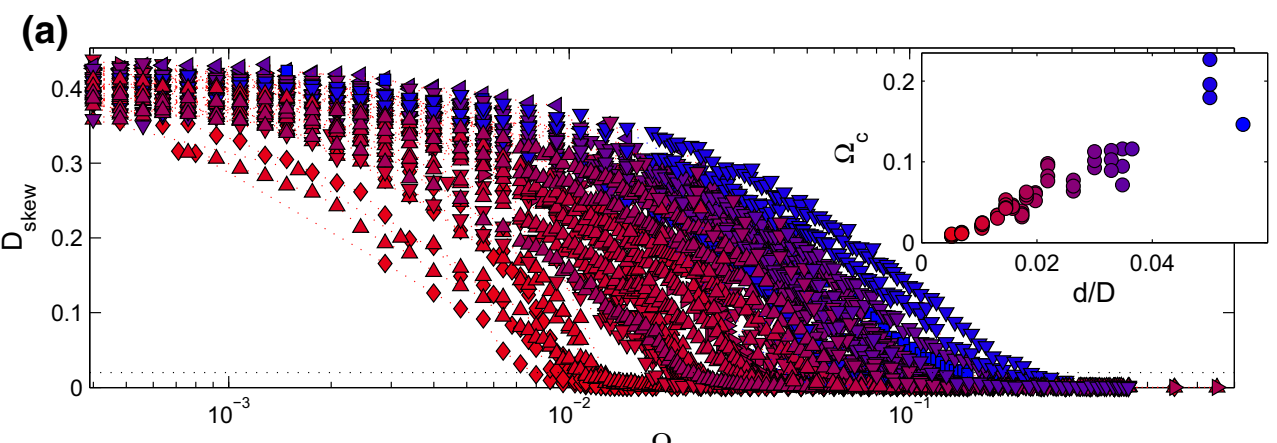

(b)

$\Omega$

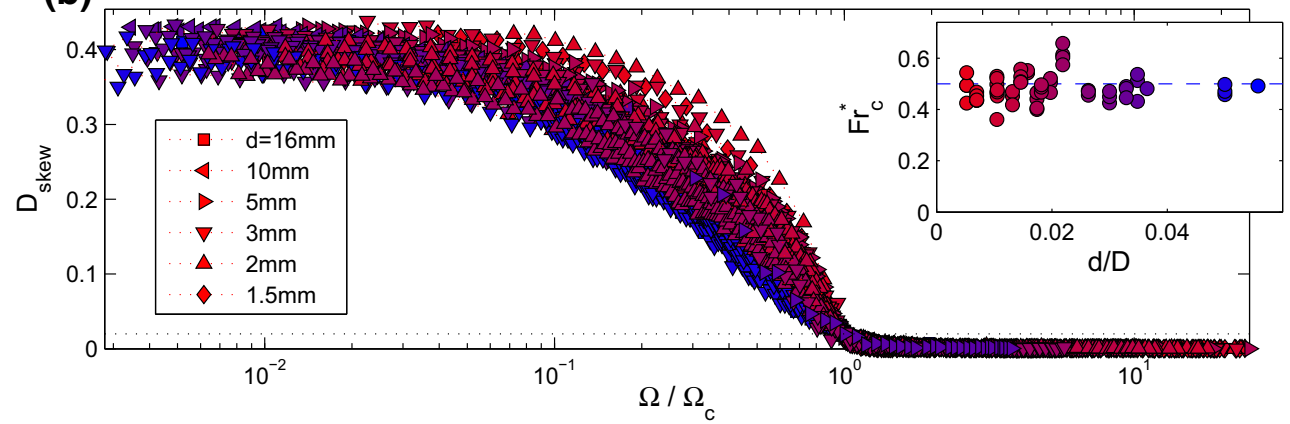

Fig. $6 D_{\text {skew }}$ data for sweeps with spheres, with the symbol conventions for particle diameter $d$ indicated. The data are plotted against $\mathbf{a}$ $\Omega$ (in $\mathrm{rad} / \mathrm{s}$ ) and $\mathbf{b} \Omega / \Omega_{c}$, where $\Omega_{c}$ is the critical rotation rate where $D_{\text {skew }}=D_{\text {crit }}$ for each sweep; here $D_{\text {crit }}=0.02$ (dotted lines). The inset of a shows $\Omega_{c}$ (in rad/s) against $d / D$. In the inset of $\mathbf{b}$, the critical rotation rates are scaled as $\mathrm{Fr}^{*}$ in (1) with $\alpha=1.1$ and $\beta=1.5$ (the dashed line shows $\left.\mathrm{Fr}^{*}=\frac{1}{2}\right)$. Symbols are color coded from red to blue according to the value of $d / D$, as indicated in the inset (color figure online)

\section{(a)}

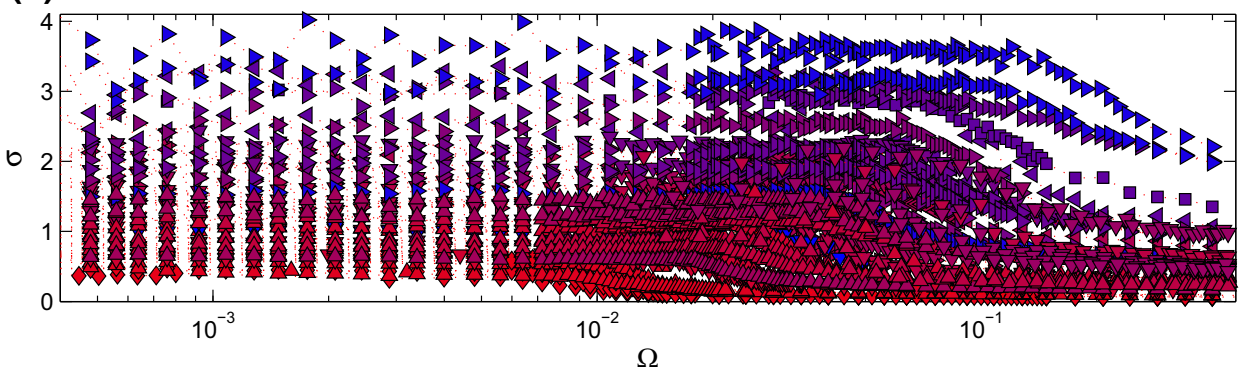

(b)

$\Omega$

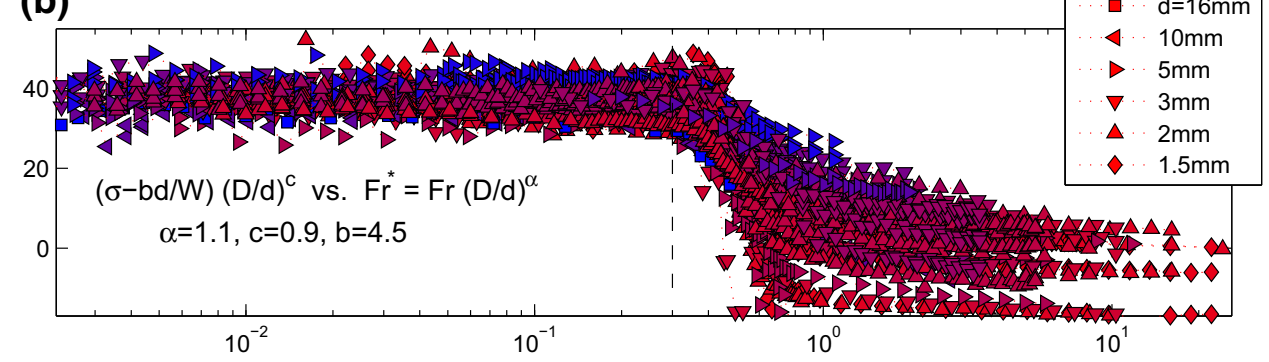

Fig. 7 Standard deviation $\sigma$ for sweeps with spheres, with the symbol conventions for $d$ indicated. a $\sigma$ is plotted against $\Omega$ (in rad/s). b The data over the slumping regime is collapsed as indicated. Symbols are color coded according to the value of $d / D$, as in Fig. 6 (color figure online)

We conclude that the upper edge of the transition is roughly given by $\mathrm{Fr}=\mathrm{Fr}^{\text {upper }}$, with

$$
\frac{\mathrm{Fr}^{\text {upper }}}{1+\beta d / W}\left(\frac{D}{d}\right)^{\alpha} \approx \frac{1}{2} \text {. }
$$

Note that the alignment of the transition by plotting $D_{\text {skew }}$ against $\Omega / \Omega_{c}$ in Fig. 6b does not collapse the entire set of sweep data onto a single curve: this diagnostic rises with rotation period for $\Omega<\Omega_{c}$ but spreads out due to an intrinsic 

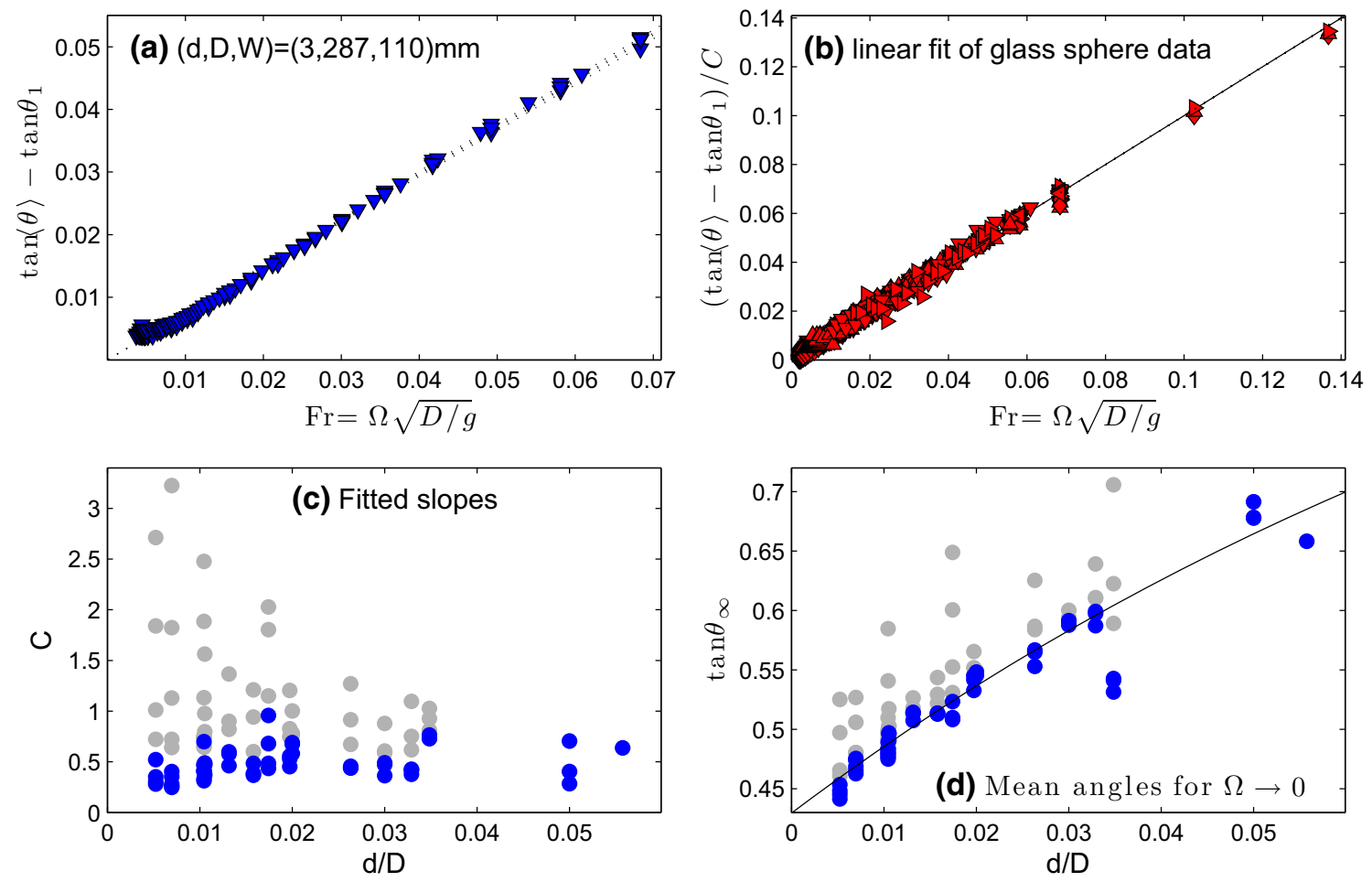

Fig. 8 a Mean angles in continuous flow $\left(D_{\text {skew }}<0.01\right)$ for $3 \mathrm{~mm}$ glass spheres in the drum with $(D, W)=(287,110) \mathrm{mm}$, plotting $\tan \langle\theta\rangle-\tan \theta_{1}$ against $\operatorname{Fr}=\Omega \sqrt{D / g}$, where $\tan \theta_{1}$ is the intercept of the linear fit (4) (dotted lines) for four different up-down sweeps. b Mean angles for all the glass sphere data, plotting $\left(\tan \langle\theta\rangle-\tan \theta_{1}\right) / C$ against Fr, where $C$ is the slope of the linear fits, using the symbol conventions

dependence on drum width ${ }^{\circledR 3}$, reflective of how episodic avalanching depends on $W$ (see Sect. 3.5).

The lower edge of the transition is better highlighted by the standard deviation $\sigma$ : this statistic becomes largely independent of rotation rate within the slumping regime; see Fig. 7a. The data for episodic avalanching can be roughly collapsed by plotting $(\sigma-b d / W)(D / d)^{c}$ against $(D / d)^{a} \mathrm{Fr}$, with $a \approx 1.1, c \approx 0.9$ and $b=4.5$. Thus, the lower edge of the transition can be conveniently located by setting $\mathrm{Fr}=\mathrm{Fr}^{\text {lower }}$ with

$\left(\frac{D}{d}\right)^{a} \mathrm{Fr}^{\text {lower }}=0.3$

Both (2) and (3) indicate that the transition migrates to zero rotation rate and disappears in the continuum limit $d / D \rightarrow 0$ (i.e. for increasingly small particles or big drums; for example, for a $1 \mathrm{~m}$ diameter drum filled with millimetersize particles, rotation periods of hours would be needed to observe episodic avalanches). Neither transition criterion is completely consistent with estimates given in previous literature $[17,24,27]$. Since the exponents $\alpha$ and $a$ are not significantly different from unity, the estimates of Liu et al. [24]

of Fig. 6. The fitting parameters $C$ and $\tan \theta_{1}$ are plotted in gray in $\mathbf{c}$ and $\mathbf{d}$. For each, the additional fits in (5) are used to suppress the dependence on drum width; the adjusted values $\left(C, \tan \theta_{1}\right)-\left(\beta_{C}, \beta_{1}\right) d / W$ are plotted as the dark (blue) points. The parameters $\beta_{1}$ are plotted later in Fig. 10. In $\mathbf{d}$, the solid line shows the fit of $\frac{1}{2}\left(\tan \theta_{\text {start }}^{\infty}+\tan \theta_{\text {stop }}^{\infty}\right)$ from Fig. 10 (color figure online)

show the same dependence on drum and particle diameter. These authors, however, also express the transition criteria in terms of the starting and stopping angles $\theta_{\text {start }}$ and $\theta_{\text {stop }}$, which they take to be material constants. In fact, these quantities depend on drum and particle geometry, as described in Sect. 3.5 and already documented in the literature (e.g. $[6,14])$.

\subsection{Continuous flow}

Mean surface slopes, $\tan \langle\theta\rangle$, during continuous flow are plotted in Fig. 8, first for $d=3 \mathrm{~mm}$ spheres in the drum with $(D, W)=(287,110) \mathrm{mm}$, and then for the whole data set. This figure illustrates that over the range of our experiments, the mean angle is well reproduced by the linear fit,

$\tan \langle\theta\rangle \approx \tan \theta_{1}+C \mathrm{Fr}$

(our attempts to collapse the data for the standard deviation $\sigma$ over the rolling regime were less successful ${ }^{\circledR 34}$ ). For the $d=$ $3 \mathrm{~mm}$ spheres, the mean slope $\tan \langle\theta\rangle$ is plotted against Froude number, subtracting off the zero-Froude number limit, $\tan \theta_{1}$; a further scaling by the slope of the linear fit, $C$, is used 
(a) $W=17 \mathrm{~mm}$

(b) $W=31 \mathrm{~mm}$

(c) $W=56 \mathrm{~mm}$

(d) $W=110 \mathrm{~mm}$
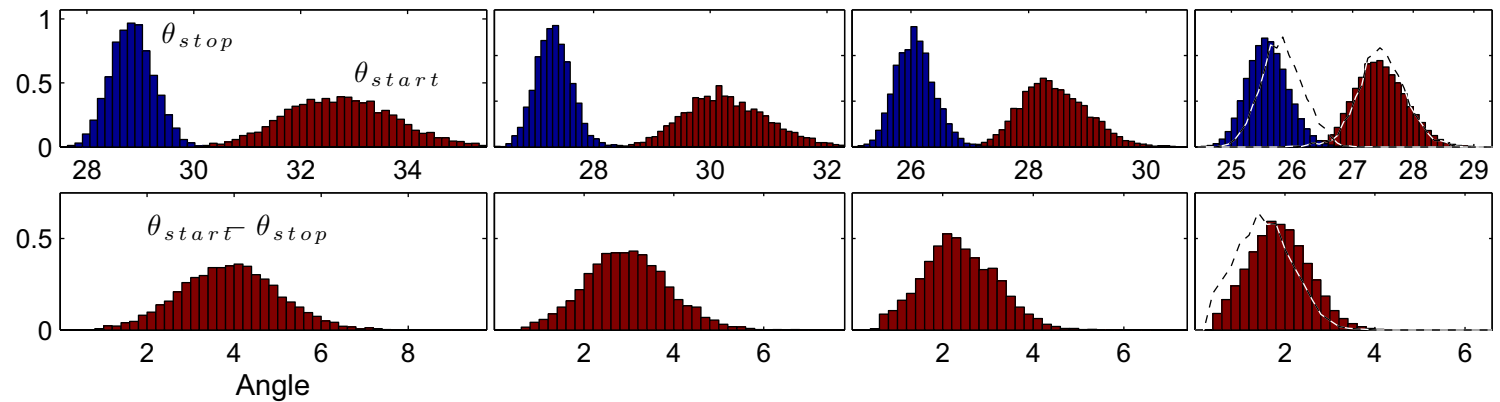

Fig. 9 Histograms of the starting and stopping angles (top row), and the avalanche amplitude (bottom row) for $d=3 \mathrm{~mm}$ glass spheres in the drum with $D=287 \mathrm{~mm}$ in the episodic regime $\left(\Omega<0.0092 \mathrm{rad} \mathrm{s}^{-1}\right)$,

for the widths indicated. In the last panels for $W=110 \mathrm{~mm}$, the dashed line shows the results for $W=205 \mathrm{~mm}$

in the plot for the entire data set. Here, the continuous flow regime is defined as $D_{\text {skew }}<D_{\text {crit }}=0.01$, which is slightly smaller than in Fig. 6, but helps to ensure that all the data are above the transition; the choice leads to a minor improvement in fits to the data but is otherwise inconsequential. Only at low rotation rates is there any discernible departure from (4), where the impending transition to episodic avalanching prompts the data to flatten out. Other than this feature, the mean slope data offer little warning of the transition. Indeed, the flattening of the mean slopes is consistent with the noisedriven emergence of incipient episodic avalanching which raises slope angles (see Fig. 2); if one were to delete any such events from the time series of $\theta(t)$, the mean surface slopes may well continue the linear trend to even lower rotation rate.

The intercepts, $\tan \theta_{1}$, and slopes, $C$, of the fits show some dependence on the drum diameter and width. We quote the values of $\theta_{1}$ for $(D, W)=(287,110) \mathrm{mm}$ in Table 1 . As shown in the lower panels of Fig. 8, much of the dependence on drum width can be suppressed by using additional fits of the form,

$C=C^{\infty}+\frac{\beta_{C} d}{W}$ and $\tan \theta_{1}=\tan \theta_{1}^{\infty}+\frac{\beta_{1} d}{W}$,

with parameters $\left(C^{\infty}, \theta_{1}^{\infty}, \beta_{C}, \beta_{1}\right)$. The adjusted mean slopes, $\tan \theta_{1}-\beta_{1} d / W$, vary in a clearer fashion with the ratio of particle to drum diameter, $d / D$ (Fig. 8c). The adjusted parameters, $C-\beta_{c} d / W$, do not vary significantly with $d / D$; the remaining scatter in Fig. 8d more likely represents differences in surface properties or dispersivity between the different spheres.

\subsection{Slumping statistics}

Statistics of episodic avalanching for the spheres are collected together in Figs. 9, 10, 11. The first picture displays histograms of the starting and stopping angles, and the avalanche amplitudes, $\Delta \theta=\theta_{\text {start }}-\theta_{\text {stop }}$, for $d=3 \mathrm{~mm}$ in the drum with $D=287 \mathrm{~mm}$ and varying width. Such angle histograms are largely independent of rotation rate, $\$ 35$ allowing the combination of all the data in the slumping regime. The histograms of $\theta_{\text {start }}$ and $\theta_{\text {stop }}$ appear Gaussian (cf. [11]), although there are hints of skewness. Aside from a change in mean, the $\theta_{\text {stop}}$-distribution appears to be independent of drum width. ${ }^{S 36}(d)$ In the widest drums, the $\theta_{\text {start }}$-distribution is similar to that for $\theta_{\text {stop }}$, but widens significantly as the drum narrows. $\$ 36(c)$ The starting and stopping angle distributions also do not remain separated but overlap in the wider drums, leading to an avalanche amplitude distribution that falls linearly to zero amplitude for $\Delta \theta \rightarrow 0,{ }^{\circledR} 36$ allowing for the persistence of arbitrarily small avalanches.

The wider spread in the starting angle distribution in the narrower drums reflects higher variability in the strength of the spheres due to confinement and, as we show later, is much less prominent for sand. One interpretation of the variability in the bridging effect is that force chains appear for some packings and not others, and more often for spheres than for sand. Moreover, isolated stress-supporting structures of this kind are more likely to inhibit the onset of flow rather than interrupt it, thus not impacting the stopping angle.

Figure 10 displays the median starting and stopping angles, averaged across the episodic regime $\left(\operatorname{Fr}(D / d)^{\alpha}<\right.$ 0.3 with $\alpha=1.1$ ). To less than one percent, the mean slope angle is equal to the average of the starting and stopping angles. The width dependence can again be suppressed using fits of the form,

$\tan \theta_{\text {start, stop }}=\tan \theta_{\text {start,stop }}^{\infty}+\beta_{\text {start,stop }} \frac{d}{W}$.

The limiting angles $\theta_{\text {start,stop }}^{\infty}$ depend on particle and drum diameter; fits to an exponential dependence on $d / D$ are included in Fig. 10a, b. The parameters, $\beta_{\text {start, stop }}$, are plotted 


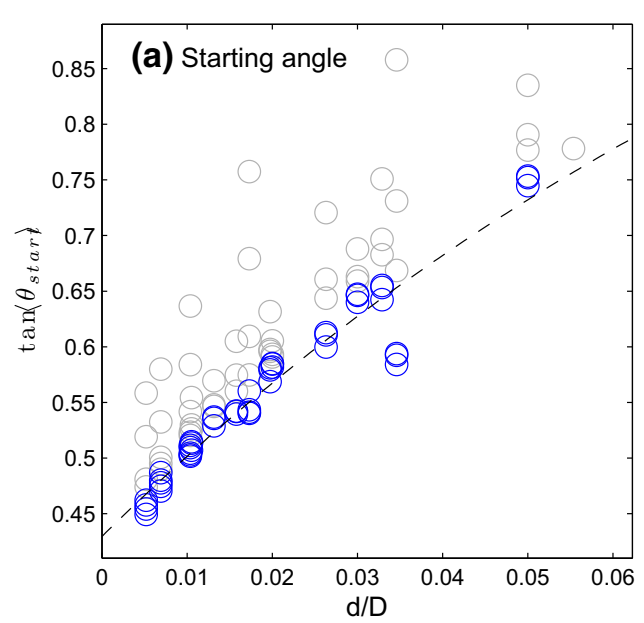

Fig. 10 The median a starting and $\mathbf{b}$ stopping angles for glass spheres, averaged over the episodic regime $\left(\operatorname{Fr}(D / d)^{a}<0.3\right)$ and plotted in gray against $d / D$. For each $d$ and $D$, the angles are fitted by $\tan \theta_{\text {start,stop }}=\tan \theta_{\text {start,stop }}^{\infty}+\beta_{\text {start,stop }} d / W$; the extrapolations to infinite width, $\tan \theta_{\text {start,stop }}-\beta_{\text {start,stop }} d / W$, are then plotted in blue. The dashed line shows $a+b_{\text {start,stop }}\left(e^{-\gamma d / D}-1\right)$
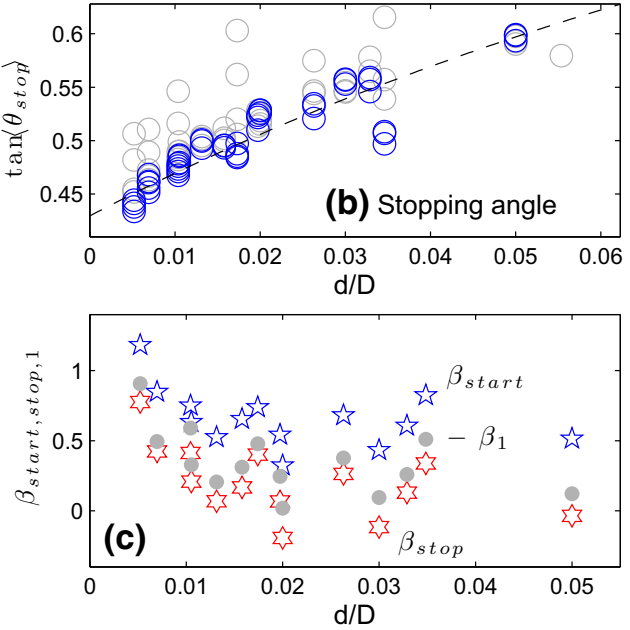

where the parameters $\left(a, b_{\text {start }}, \gamma\right)$ are chosen by a least-squares fit to the extrapolated starting angle data, and $b_{\text {stop }}$ using a fit to the extrapolated stopping angle data. Panel $\mathrm{c}$ plots $\beta_{\text {start,stop }}$ against $d / D$ (blue pentagrams and red hexagrams); the gray dots are the fitting parameters $\beta_{1}$ from Fig. 8 (color figure online) (a) $\mathrm{W}=17 \mathrm{~mm}$

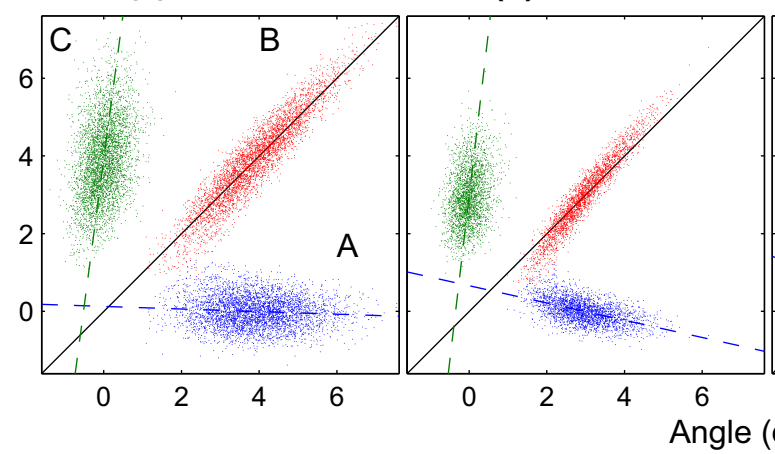

(c) $W=56 \mathrm{~mm}$

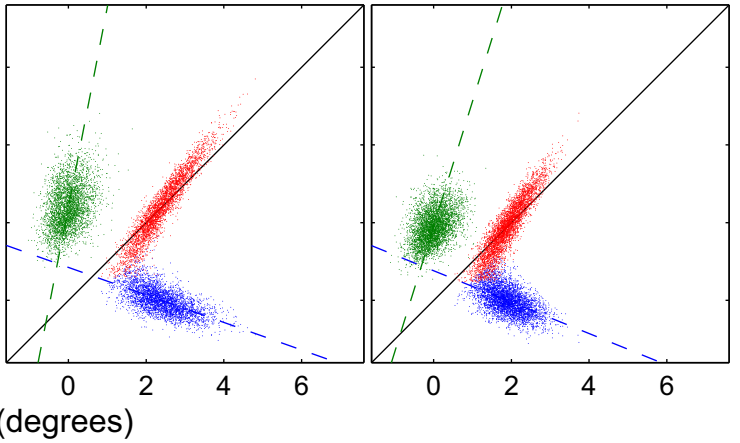

Fig. 11 Scatter plots of starting and stopping angles for $d=3 \mathrm{~mm}$ spheres in drums with $D=287 \mathrm{~mm}$ and the widths indicated, taken over the entire episodic avalanching regime with $\Omega<0.005 \mathrm{rad} \mathrm{s}^{-1}$. In each case, three sets of data are shown: $A$ plots $\theta_{\text {start }}-\left\langle\theta_{\text {stop }}\right\rangle$ on the $x$-axis against the subsequent $\theta_{\text {stop }}-\left\langle\theta_{\text {stop }}\right\rangle$ on the $y$-axis; $B$ plots $\theta_{\text {start }}-\left\langle\theta_{\text {stop }}\right\rangle$

in Fig. 10c and are consistent with the fitting parameters $\beta_{1}$ used for the continuous flow data in Fig. 8 (which are also plotted), and values reported previously $[6,14]$.

Figure 11 presents "scatter" plots of the starting and stopping angles and the avalanche amplitude. The clearest feature of these plots is the tight connection between $\theta_{\text {start }}$ and the ensuing amplitude $\Delta \theta=\theta_{\text {start }}-\theta_{\text {stop }}$ (data labeled $B$ ); i.e. for a given avalanche, the higher starting angle, the larger the avalanche (cf. Caponeri et al. [4]). As pointed out by Fischer et al. [11], the data also suggest a weak negative correlation between $\theta_{\text {start }}$ and the subsequent $\theta_{\text {stop }}($ data labeled $A$ ); the higher the start, the lower the stop. The weakness of this correlation implies that intrinsic noise and dissipation during
( $x$-axis) against the ensuing avalanche amplitude $\Delta \theta=\theta_{\text {start }}-\theta_{\text {stop }}(y$ axis); $C$ plots $\theta_{\text {start }}-\left\langle\theta_{\text {stop }}\right\rangle$ ( $x$-axis) against the preceding $\theta_{\text {stop }}-\left\langle\theta_{\text {stop }}\right\rangle$ ( $y$-axis). The dashed lines show least-squares linear fits to $A$ and $C$, and the solid line is the diagonal

flow are sufficient to wipe out much of the memory of the initiation of the avalanche.

Despite what is commonly assumed in the literature (e.g. [33]), the plots also suggest a comparable correlation of $\theta_{\text {start }}$

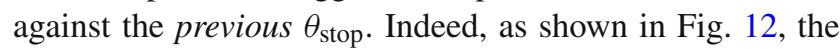
magnitude of the average correlation of $\theta_{\text {start }}$ with either the subsequent or previous $\theta_{\text {stop }}$ is about 0.25 over the episodic range for the narrowest drums (the correlations are again largely independent of $\Omega$ in this regime ${ }^{(37}$ ). The correlations become stronger as the drum is widened, before falling off somewhat for the widest drums, perhaps because of the axial decorrelation of successive avalanches. 

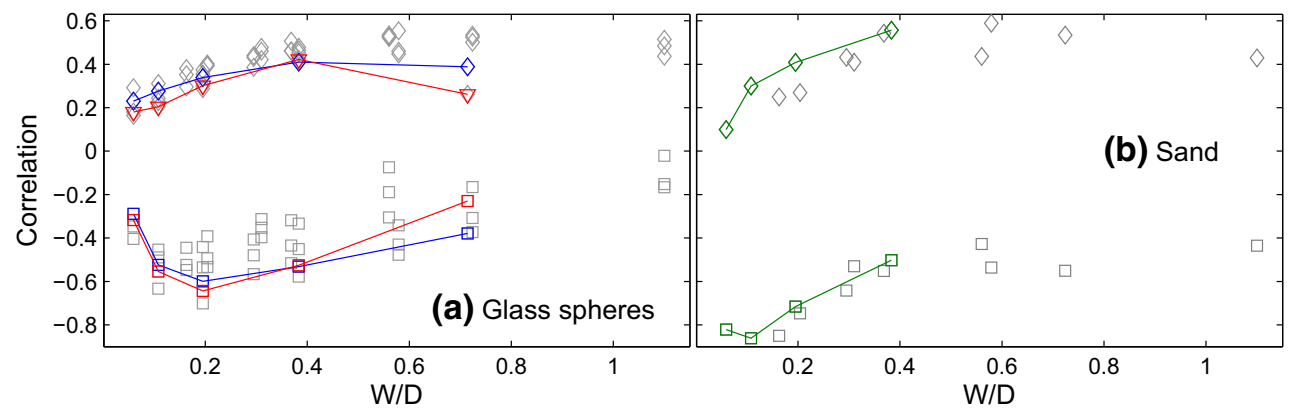

Fig. 12 Correlation coefficients between $\theta_{\text {start }}$ and the following $\theta_{\text {stop }}$ (squares) and the previous $\theta_{\text {stop }}$ (diamonds) plotted against $W / D$ for a all the glass spheres and $\mathbf{b}$ sand, averaged over the episodic avalanch- ing regime. In a, the data for the big drum $D=287 \mathrm{~mm}$ are connected by solid lines for $d=2 \mathrm{~mm}$ (red) and $3 \mathrm{~mm}$ (blue). In $\mathbf{b}$, the data for $D=287 \mathrm{~mm}$ are connected by a (green) solid line (color figure online)

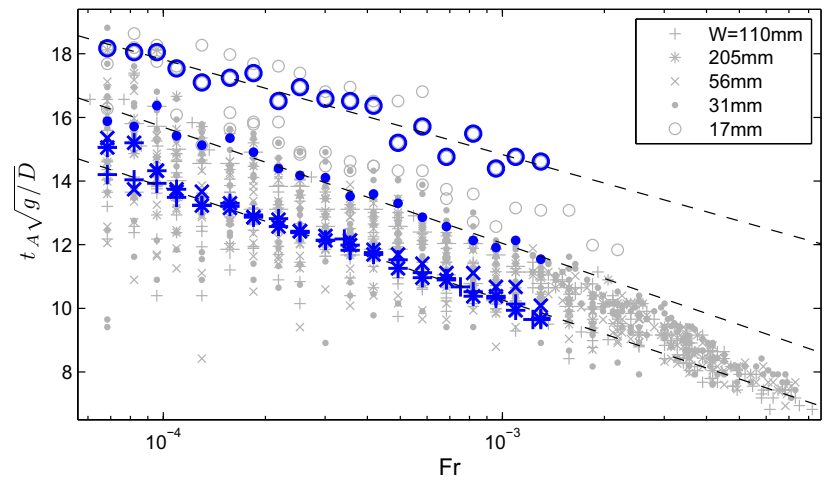

Fig. 13 Scaled median avalanches durations $t_{A} \sqrt{g / D}$ against Fr for all the glass sphere data in the episodic regime (gray), with symbols corresponding to different drum widths as indicated. The dark (blue) symbols display $3 \mathrm{~mm}$ data for $D=289 \mathrm{~mm}$; the dashed lines are linear fits of $t_{A} \sqrt{g / D}$ with $\log$ Fr for the drums with $W=110,31$ and $17 \mathrm{~mm}$ (color figure online)

A correlation between $\theta_{\text {start }}$ and the preceding $\theta_{\text {stop }}$ is possible when the packing of material at the termination of an avalanche, reflecting to some degree the $\theta_{\text {stop }}$, affects subsequent failure, i.e. $\theta_{\text {start }}$. However, each avalanche uncovers fresh material in the upper parts of the drum, with a packing set by a much earlier collapse. Nevertheless, for spheres, failure does not always occur first at the top of the slope but can be elsewhere along the surface (Sect. 3.1), where the packing may have been set by the previous avalanche.

\subsection{Mean avalanche durations and profiles}

Figure 13 displays the mean avalanche duration, $t_{A}$, for glass spheres, defined as the average interval between the starting and stopping angles. This figure illustrates the curious result that the avalanche duration grows logarithmically as the rotation rate is decreased. There is also a modest but systematic dependence of $t_{A}$ on drum width, consistent with the idea that sidewall friction slows and thereby prolongs avalanching.
The logarithmic growth of $t_{A}$ disagrees with earlier work $[4,11,12]$ which reports avalanche durations that are independent of rotation rate. In agreement with this work, however, the scaled duration $t_{A} \sqrt{D / g}$ does not vary strongly with drum or particle diameter. Caponeri et al. [4] base their estimate of avalanche duration on the extrema of time series of the lower edge of the granular surface; given the nearly linear slope of the surface, this is similar to our measurement $t_{A}$. Fischer et al. [11] estimate durations using a Gaussian fit to the profile of individual avalanches.

To understand this blend of agreement and disagreement, we examine the avalanche structure in more detail. Figure 14 shows the mean scaled avalanche profile, obtained by averaging, over all avalanches during a particular experiment, the time series of

$\Phi(\tau)=\Phi\left(t-t_{m}\right)=\frac{\theta(t)-\theta_{\text {stop }}}{\theta_{\text {start }}-\theta_{\text {stop }}}$

where $\theta\left(t_{m}\right)=\frac{1}{2}\left(\theta_{\text {start }}+\theta_{\text {stop }}\right)$. This procedure suppresses the differences between avalanches, furnishing a smooth curve characteristic of the dynamics (cf. [11]). As shown in Fig. 14, the core of the profile remains largely unchanged as the rotation rate decreases. More significant is the change in the profile's maxima and minima, which become very flat and broad for low $\Omega$. Avalanches therefore last longer for higher rotation period because they take more time to begin and end, not from any change in the time required for the surface angle to fall once motion is underway. The latter can be quantified by measuring $t_{1 / 2}$, the time required for $\Phi(t)$ to decrease from $3 / 4$ to $1 / 4$, which is more comparable to the characteristic avalanche duration defined by Fischer et al. Figure $14 d$ plots both $t_{1 / 2}$ and $t_{A}$ against Fr; $t_{1 / 2}$ does indeed become constant within the slumping regime. Note that, at the higher rotation rates, the mean avalanche profiles also display the preceding and ensuing avalanches, a feature resulting from a well-defined mean avalanche spacing (see below). 

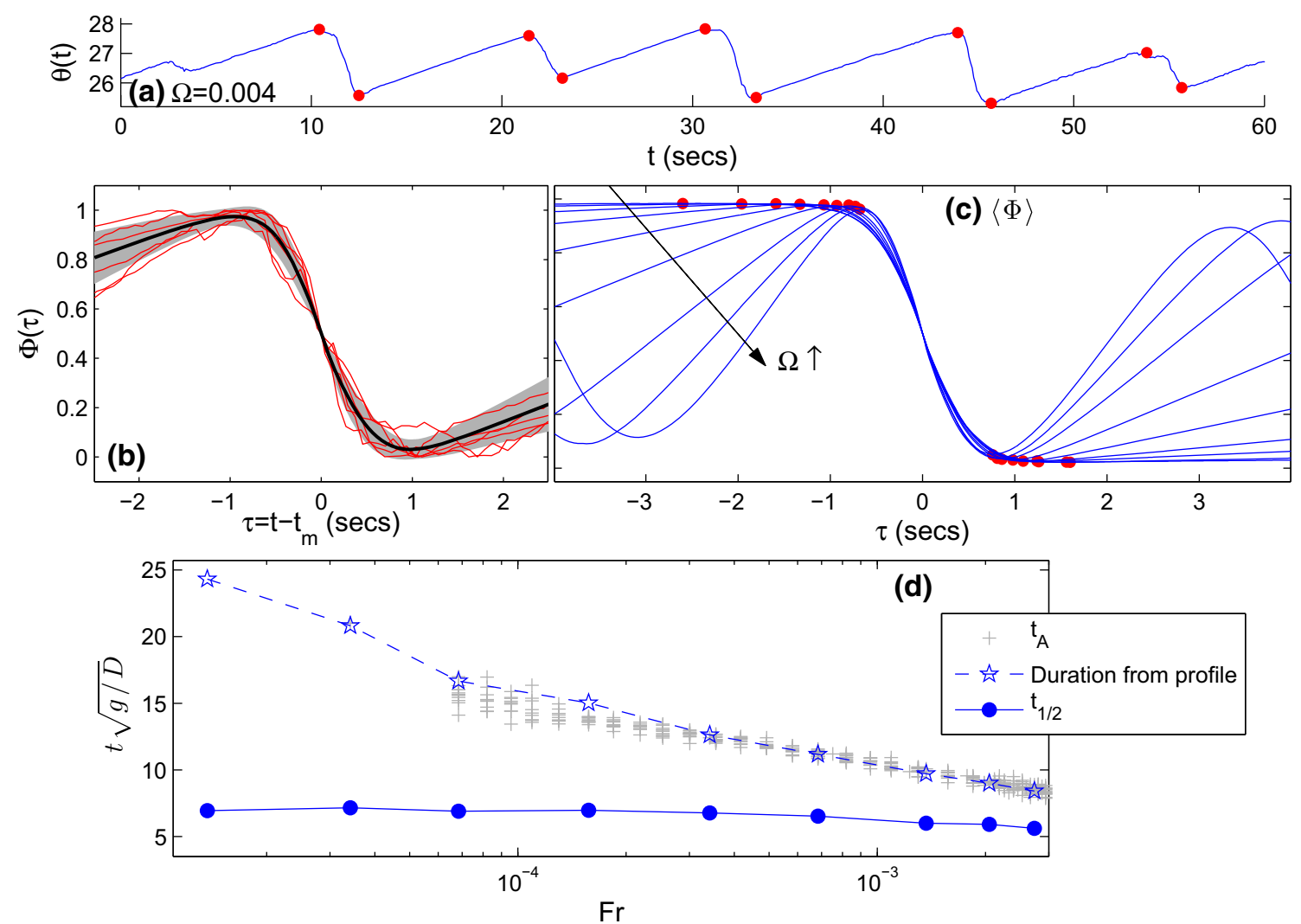

Fig. 14 Avalanche structure for $(D, W)=(287,110) \mathrm{mm}$ and $3 \mathrm{~mm}$ spheres. a Time traces of surface angle at $\Omega=0.004 \mathrm{rads}^{-1}$, with the identified start and stop angles shown; the small avalanche near $t=3 \mathrm{~s}$ is not identified due to the clipping tolerances in the extremum search. b The mean scaled avalanche profile, obtained by averaging $\Phi\left(\tau=t-t_{m}\right)$ in (7). Also shown are six sample avalanches (red) and

\subsection{Spectra}

In Fig. 15, we show power spectra for $3 \mathrm{~mm}$ spheres in the drum with $(D, W)=(287,110) \mathrm{mm}$. At the lowest rotation rates (the top curves), there is a broad peak at an angular frequency $\omega$ that is a few hundred times $\Omega$. The spectra level out at lower frequency, and then fall off algebraically at higher frequency, first with a dependence $\omega^{-2}$, then steepening up to $\omega^{-4}$ and finally flattening off like $\omega^{-1}$-noise. As one approaches the slumping-to-rolling transition, the dominant peak sharpens and its harmonic becomes visible. The peak almost disappears in the continuous flow regime, leaving a flat red spectrum. The frequency of the dominant peak of the power spectrum is plotted against rotation rate in Fig. 16 for $(d, D)=(3,287) \mathrm{mm}$ and varying width.

The spectra are contaminated by additional peaks at the characteristic frequency $\omega_{m}$ of the stepper motor and some of its harmonics. These become visible at high rotation rate, occasionally taking over the dominant frequency. Further high-frequency peaks at $\omega_{s}=8000 \omega_{m}$ and its harmonics the mean plus or minus the standard deviation (shaded region). $\mathbf{c}$ Nine mean avalanche profiles for $W=110 \mathrm{~mm}$ at the rotation rates indicated in $\mathbf{d}$; the maxima and minima are indicated. $\mathbf{d}$ The avalanche duration defined as the time between the maximum and minimum of the mean scaled avalanche profiles. The characteristic time $t_{1 / 2}$ is also shown, as well as direct measurements of $t_{A}$ (gray crosses) (color figure online)

correspond to the steps of the motor. Although the extraneous peaks appear in the spectra, they have a clear origin and there is no suggestion that they influence the granular dynamics in general.

Over the episodic avalanching regime, most features of the power spectrum can be reproduced by a relatively simple stochastic model (Appendix C). In brief, one assumes that the starting and stopping angles and the avalanche duration $t_{A}$ are independent random variables (i.e. ignoring the correlations exposed in Sect. 3.5), and that the signal for the surface angle is composed of two linear pieces: a rise at rate $\Omega$ during solidbody rotation between $\theta_{\text {stop }}$ and $\theta_{\text {start }}$, then a linear collapse of duration $t_{A}$ between $\theta_{\text {start }}$ and the next $\theta_{\text {stop. }}$. The spectrum of this signal features a dominant frequency of

$\omega_{*}=\frac{2 \pi \Omega}{\left\langle\Delta \theta+\Omega t_{A}\right\rangle}$

(cf. [7]). This prediction is included in Fig. 16, using the linear fits of $t_{A}$ with $\log$ Fr taken from Fig. 13. 


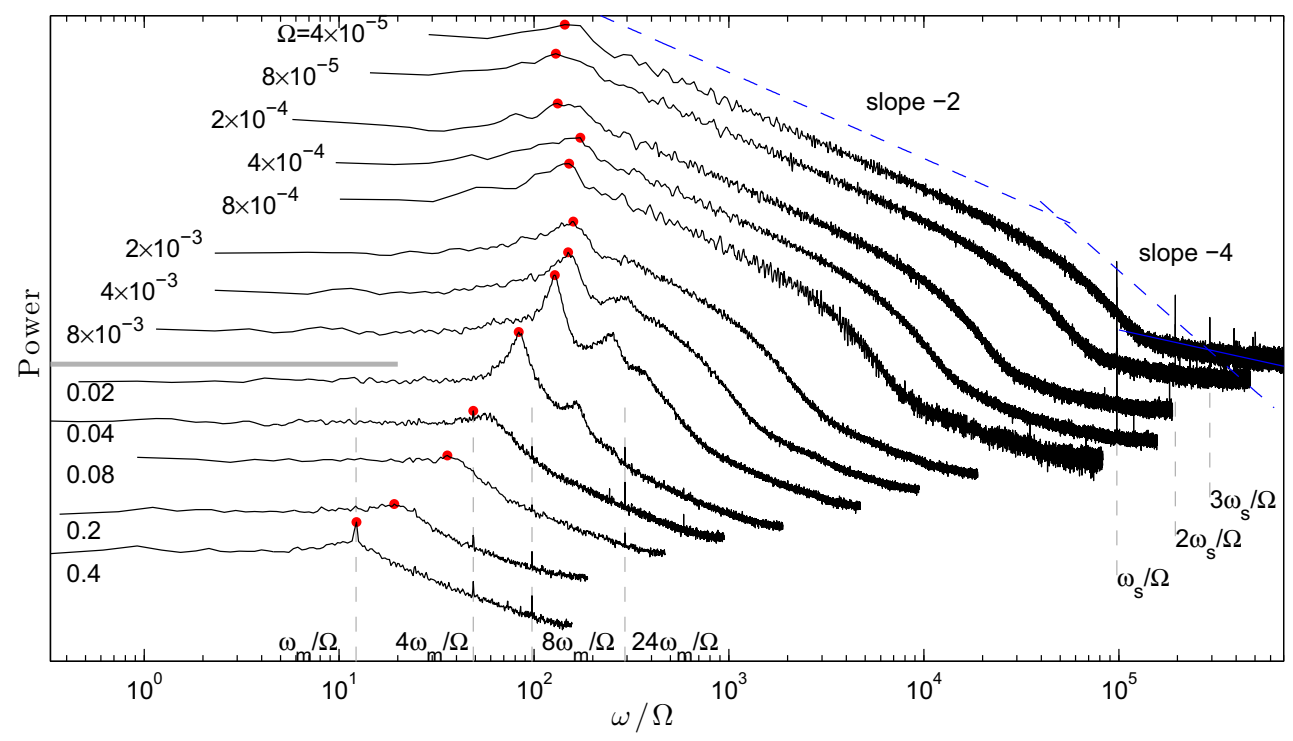

Fig. 15 Power spectra for $3 \mathrm{~mm}$ spheres in the drum with $(D, W)=$ $(287,110) \mathrm{mm}$, plotted against $\omega / \Omega$ for the rotation rates indicated, where $\omega$ is the angular frequency (with both $\Omega$ and $\omega$ in $\mathrm{rad} / \mathrm{s}$ ). The spectra are offset for clarity and the dominant peak is identified. The ver- tical dashed lines show the additional frequencies $\omega_{m}, \omega_{s}=8000 \omega_{m}$ and some of their harmonics. The transition is indicated by the gray horizontal line. The inclined dashed lines indicate slopes of -2 and -4

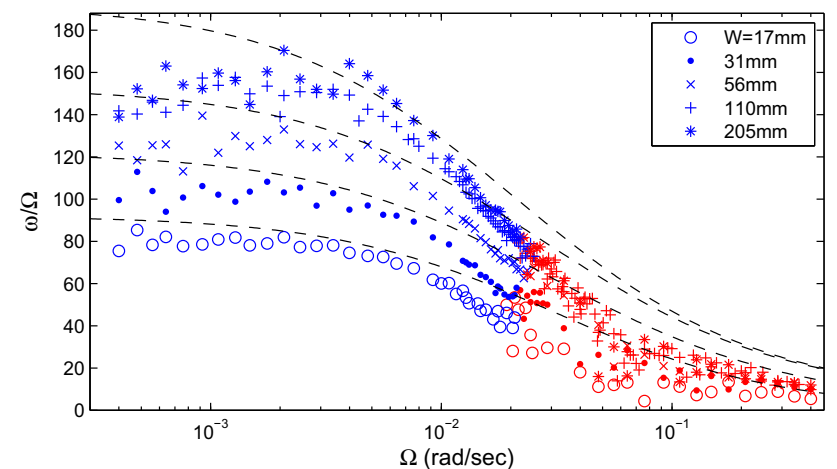

Fig. 16 The frequency of the dominant peak in the power spectra for $d=3 \mathrm{~mm}$ spheres in the big drum with the widths indicated, plotting $\omega / \Omega$ against $\log \Omega$. The blue (red) symbols indicate episodic avalanching (continuous flow). The dashed lines are the fits in (8) for $W \leq 110 \mathrm{~mm}$ (color figure online)

In addition to the main peak, the spectrum of the synthetic signal is flat at low frequency where avalanches look like a random succession of impulses (cf. [4]). For $\omega_{*} \ll \omega \ll$ $\left\langle t_{A}\right\rangle^{-1}$, the solid-body rise of the signal becomes resolved, but not the relatively rapid avalanches, so the signal resembles a sawtooth with the spectrum falling like $\omega^{-2}$. Finally, for $\omega \gg t_{A}^{-1}$, the continuity of the signal over the avalanches is resolved and the spectrum then falls like $\omega^{-4}$. With an avalanche duration of a few seconds (Fig. 13), the cross-over in the tail of the spectrum occurs for $\omega \sim t_{A}^{-1} \sim 1 \mathrm{rad} / \mathrm{s}$, in rough agreement with the switch in scalings of the top curves in Fig. 15.

\section{Sand}

\subsection{Avalanche phenomenology for sand}

To explore how the dynamics changes when we switch to aspherical particles, we performed up-down sweeps with sand. Sample time series of the surface angle for different rotation rate are shown in Fig. 17. There is a much sharper switch in behavior at the slumping-to-rolling transition than for spheres, and the episodic avalanching is much more regular, producing an almost periodic signal.

The space-time plots in Fig. 18 illustrate how the avalanche dynamics in sand is also quite different from the glass spheres: recognizable fronts arise that control the avalanches, as noted previously [34]. A first front appears near the top end of the pile and propagates down to the lower end, activating the collapse. Once the advancing front reaches the bottom of the free surface, it triggers a retreating front which propagates back up the surface to the upper end, switching off the flow. The starting and stopping positions of the avalanches are consequently always near the top corner of the granular surface $^{(32}$. Neither front features in the dynamics of the spheres (cf. [11]).

\subsection{Sweeps, intermittency and hysteresis}

For both the biggest and smallest drums with $(D, W)=$ $(287,110)$ and $(100,31) \mathrm{mm}$ a smooth transition with clear intermittent switching between phases of slumping and 


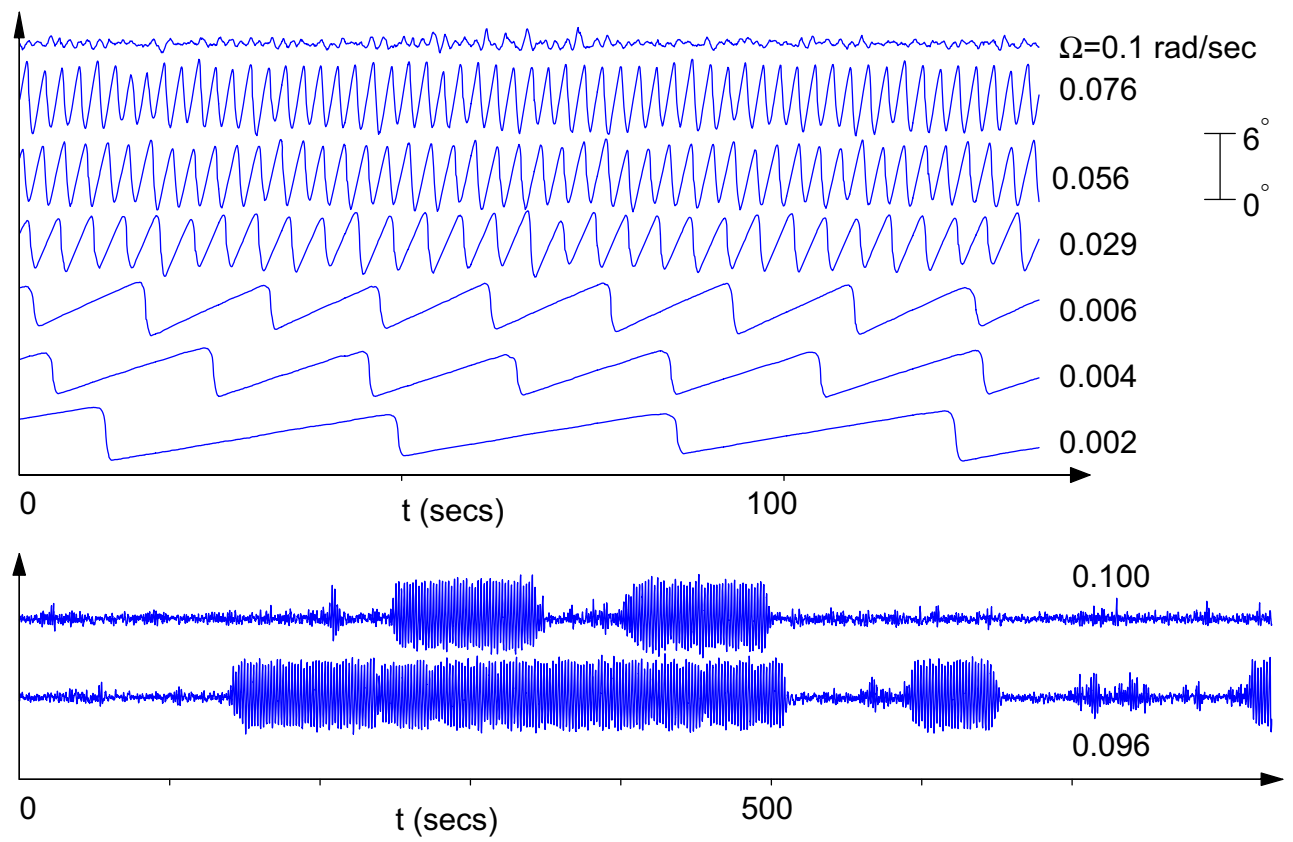

Fig. 17 Sample time traces of $\theta(t)$ for the sand in the drum with $(D, W)=(287,110) \mathrm{mm}$ at the rotation rates indicated. The two time traces in the lower panel show intermittent behavior over the transition

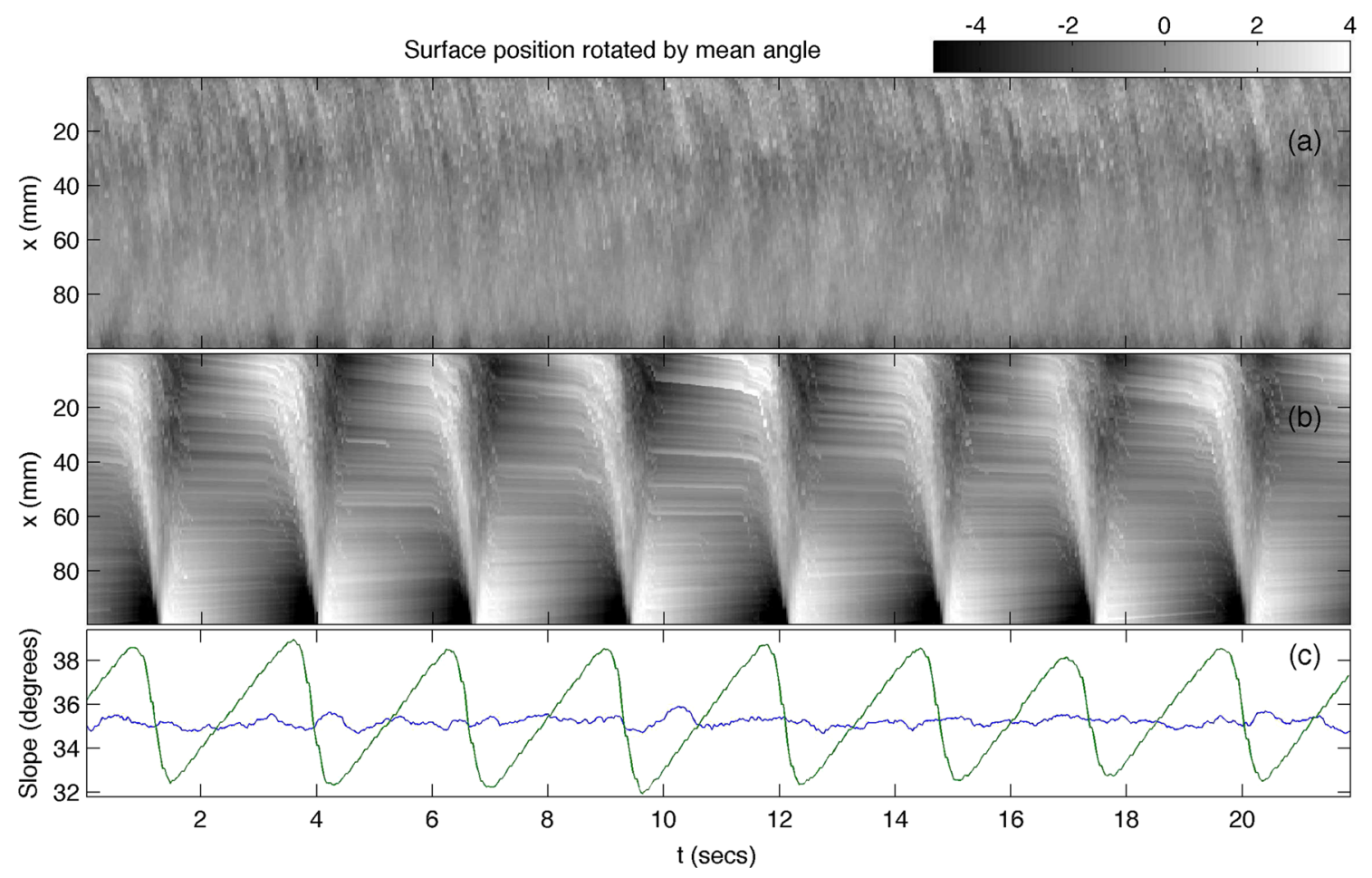

Fig. 18 A similar picture to that in Fig. 4, but for the sand $(D, W)=(137,86) \mathrm{mm}$ with $\mathbf{a} \Omega=0.27 \mathrm{rad} \mathrm{s}^{-1}$ and $\mathbf{b} \Omega=0.06 \mathrm{rad} \mathrm{s}^{-1}$

rolling is again evident (see the lower traces in Fig. 17 and the sweep data in Fig. 19b). However, for all the other drums the transition was different, occurring via a sudden jump from rolling to slumping or vice versa. Moreover, the jump from slumping to rolling occurred at higher rotation rates than the jump from rolling to slumping, leading to hysteresis in the sweep data; see Fig. 19a. Note that multiple sweeps focusing on the transition are included in this figure; these demonstrate how the transitions between the two states do not occur at a single rotation rate but at seemingly random values of 


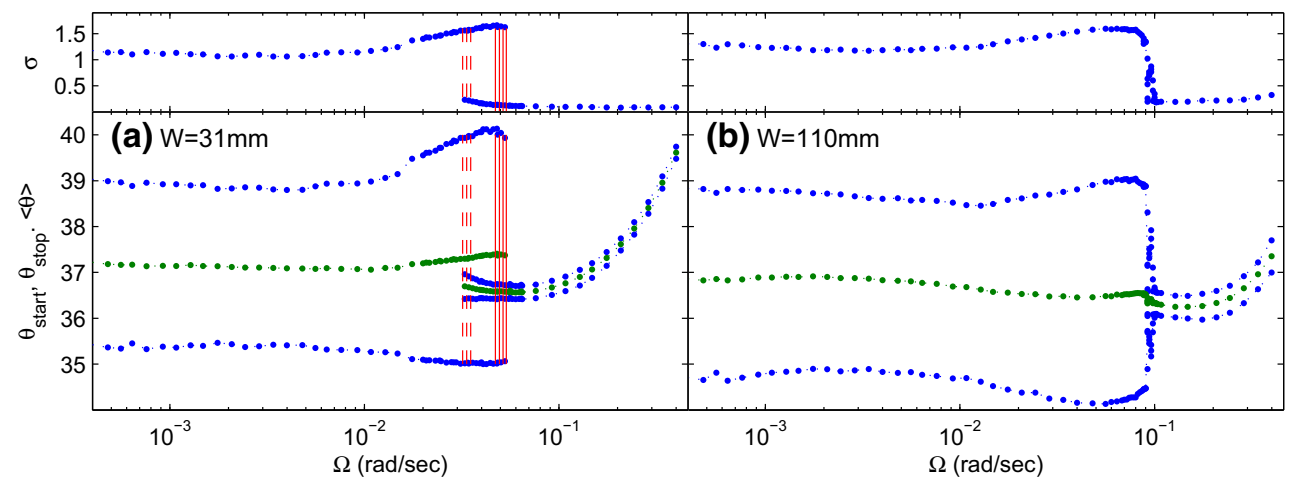

Fig. 19 Up-down sweeps for sand in the big drum with $D=289 \mathrm{~mm}$, showing angle data and standard deviation plotted against $\Omega$ for the widths indicated. The sweeps include multiple repeated runs focusing around the transition; in a the switches between slumping to rolling arise for several rotation rates, as indicated by the vertical red dashed and solid lines (marking the switch to slumping or rolling, respectively).

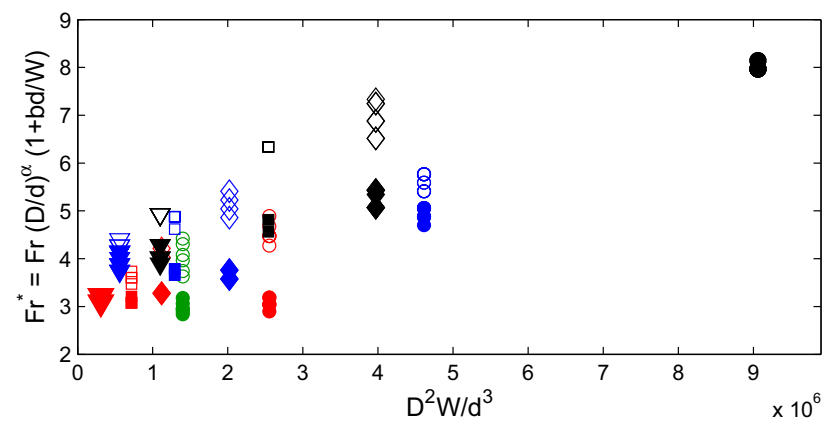

Fig. 20 Scaled transitional Froude numbers, $\operatorname{Fr}^{*}=\operatorname{Fr}(D / d)^{\alpha}(1+$ $b d / W$ ), against $D^{2} W / d^{3}$, for $\alpha=1.1$ and $b=1.5$ (see Fig. 6). Open (filled) symbols refer to the transition from slumping to rolling (rolling to slumping), with the conventions: $D=289 \mathrm{~mm}$-circles, $190 \mathrm{~mm}$ - diamonds, $152 \mathrm{~mm}$ - squares, and $100 \mathrm{~mm}$ - triangles, and $W=110 \mathrm{~mm}$-black, $56 \mathrm{~mm}$-blue, $31 \mathrm{~mm}$-red, $17 \mathrm{~mm}$-green (color figure online)

$\Omega$ spread over ranges that are narrower than the window of hysteresis.

A summary of the transitional Froude numbers for sand (as defined by $D_{\text {skew }}=0.02$ ) is provided in Fig. 20, which plots the data against the volume ratio $D^{2} W / d^{3}$ and scales Fr as for the glass spheres in Fig. 6. The transition evidently occurs at rather higher rotation rates than predicted by (2). The scaling of the Froude number is also unable to align the data for either the intermittent drums or the two transitions bordering the region of hysteresis. Thus, the scalings observed for glass spheres do not work for sand. Partly responsible for this discrepancy is that the transition in sand depends significantly on drum width, unlike for spheres. This result is made more surprising by the fact that the statistics of rolling and slumping do not appear to depend significantly on the drum width (see below), again in contrast to spheres.

It is also not clear why the window of hysteresis opens in between our largest and smallest drums. Conceivably,
The data vary more strongly with $\Omega$ within the slumping regime than for spheres, at least for $\Omega>0.01 \mathrm{rad} \mathrm{s}^{-1}$, reflecting how the episodic avalanching occurs at higher rotation rates where drum speeds are no longer much smaller than flow speeds during an avalanche (color figure online)

enhanced fluctuations with fewer particles might wash out a hysteretic transition in the smallest drum; perhaps the opportunity for spatial decorrelation triggers additional perturbations to rationalize why the biggest drum shows an intermittent transition. Either way, the window of hysteresis likely closes at the two extremes due to an increase in the effective system noise.

Curiously, the mean angles for continuous flow in sand show a much more prominent upturn as one approaches transition. This is illustrated in Fig. 21, which plots the mean surface angles offset by the minimum slope and plotted against Froude number scaled by the value $\mathrm{Fr}_{\min }$ where the minimum is attained. This way of plotting the data removes much of the variation between the different drum geometries; the minimum slope depends on drum diameter but not obviously on its width, whereas $\mathrm{Fr}_{\text {min }}$ depends on both (see Fig. 21b, c). In the simple model of [4] an upturn in the continuous flow solution arises from the non-monotonic dependence of the friction law, and destabilizes that equilibrium to trigger the switch to slumping. No such instability is manifest in the experiments, with the upturn of the mean angle occupying a significant range of rotation rates characterized by robust continuous flow. This feature may explain earlier observations [32] that the mean angle increases like the square of the rotation rate, which is otherwise not supported by the data.

\subsection{Avalanche statistics and profiles for sand}

Figure 22 shows slumping statistics for sand; the corresponding starting-stopping-angle correlations are compared with results for glass spheres earlier in Fig. 12. Figure 22 illustrates how the dependence of the starting angle distribution on drum width is much less marked for sand. Moreover, in 
(a)

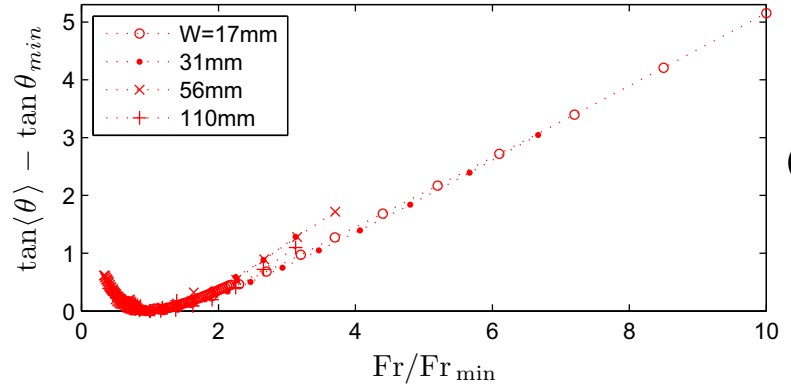

(b)

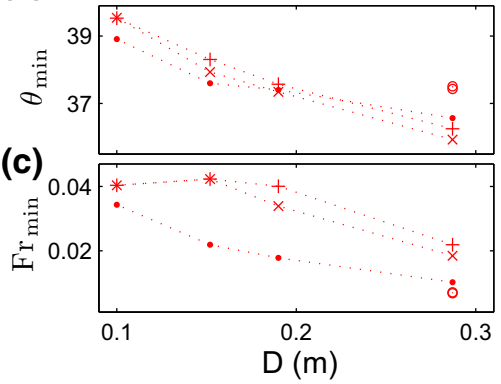

Fig. 21 Mean slopes in the rolling regime for sand, plotting $\mathbf{a} \tan \theta-\tan \theta_{\min }$ against $\mathrm{Fr} / \mathrm{Fr}_{\text {min }}, \mathbf{b} \tan \theta_{\min }$ against $D$, and $\mathbf{c} \mathrm{Fr}_{\text {min }}$ against $D$, with the symbol convention for drum width indicated

(a) $\mathrm{W}=17 \mathrm{~mm}$

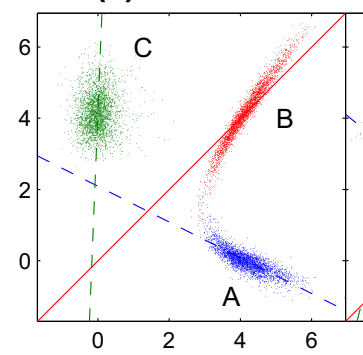

(b) $\mathrm{W}=31 \mathrm{~mm}$

(c) $W=56 \mathrm{~mm}$

(d) $W=110 \mathrm{~mm}$
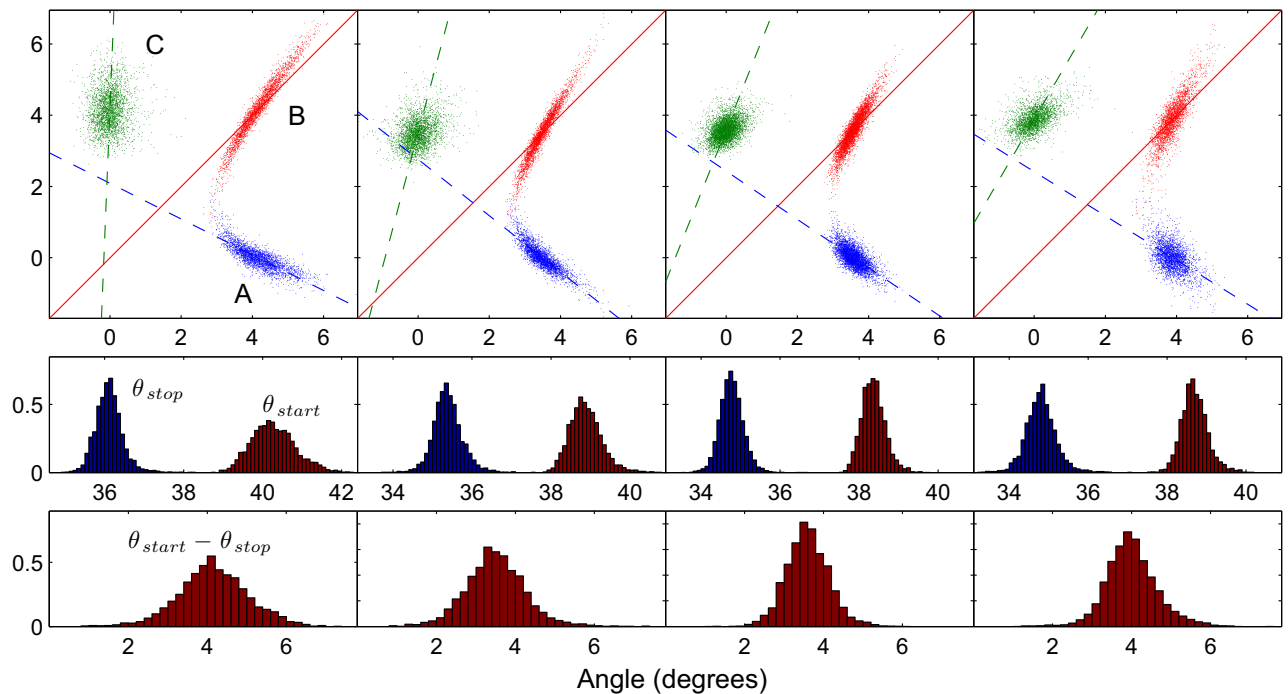

Fig. 22 Avalanche statistics for sand in the drum with $D=287 \mathrm{~mm}$ and the widths indicated, averaged over the episodic regime $(\Omega<$ $\left.0.01 \mathrm{rad} \mathrm{s}^{-1}\right)$. Top row: scatter plot of $\theta_{\text {start }}-\left\langle\theta_{\text {stop }}\right\rangle$ ( $x$-axis) against (on the $y$-axis) the following $\theta_{\text {stop }}-\left\langle\theta_{\text {stop }}\right\rangle$ ( $A$; blue dots), the avalanche amplitude $\theta_{\text {start }}-\theta_{\text {stop }}(B ;$ red dots $)$, and the preceding $\theta_{\text {stop }}-\left\langle\theta_{\text {stop }}\right\rangle$ ( $C$; green dots). The dashed lines show least-squares linear fits to $A$ and $C$, and the solid line is the diagonal. Lower row: histograms of the starting and stopping angles (color figure online) the wider drums, the starting and stopping angles remain well separated, furnishing a more Gaussian-like amplitude distribution that favors regular avalanching rather than collapses of arbitrarily low amplitude. The sand amplitude distribution is consequently sensitive to drum diameter but has no clear dependence on width ${ }^{\circledR} 38,39$, unlike that for spheres.

The correlations between the starting and stopping angles are also different for sand (Fig. 12b). In the narrow drums, $\theta_{\text {start }}$ is poorly correlated with the previous $\theta_{\text {stop }}$, consistent with the observation that the avalanches begin at the top of the drum, where fresh material has been exposed by the previous avalanche and the packing is relic from the distant past. The starting angle, however, is strongly negatively correlated with the subsequent stopping angle, and so dissipation and dynamical noise during flow cannot erase the memory of avalanche initiation. Widening the drum strengthens the cor- relation of $\theta_{\text {start }}$ with the preceding $\theta_{\text {stop }}$ whilst reducing its

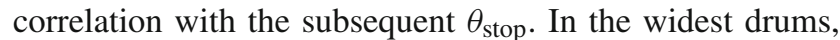
$\theta_{\text {start }}$ is roughly equally correlated with both (coefficients of about \pm 0.5 ). Evidently, the wider drum features greater dynamical noise that suppresses the memory of initiation; the correlation with the previous start is less straightforward to understand.

Sample avalanche profiles for sand are shown in Fig. 23. Once again $t_{A}$ increases logarithmically with rotation period due to the increasing time taken to initiate and terminate each avalanche. For wider drums, the time needed to start the avalanche dominates, whereas the time taken to end the event is more critical in narrower drums. The avalanche profiles contain more structure than their relatives for spheres (cf. Fig. 14). Most noticeable is the kink near the midpoint of the profile, which is caused by the two fronts that switch flow 

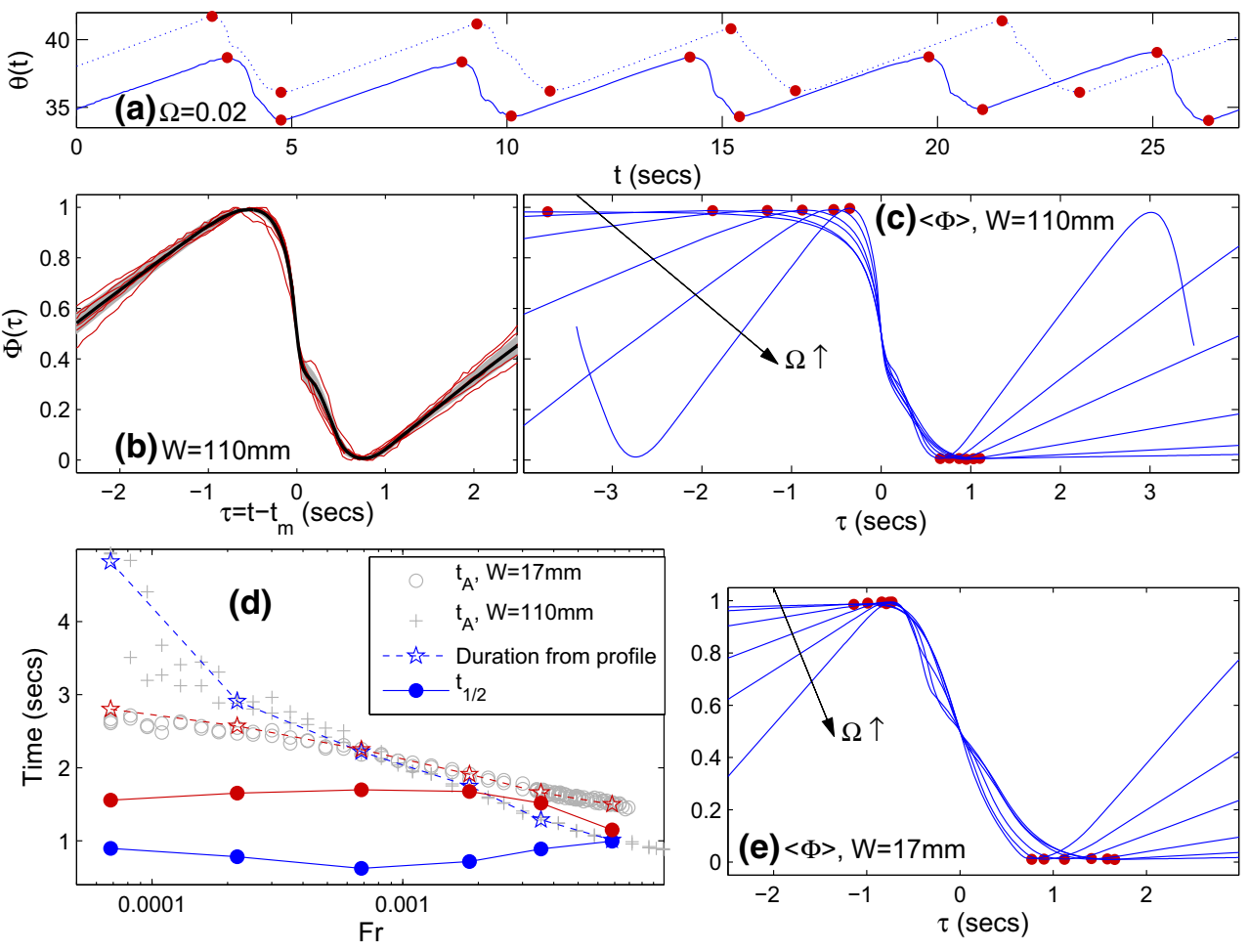

Fig. 23 a Time series of surface angle for sand in the $D=287 \mathrm{~mm}$ drum with $W=110 \mathrm{~mm}$ (solid) and $17 \mathrm{~mm}$ (dotted). b Mean scaled avalanche profile for the wider drum, plus or minus the standard deviation and six sample avalanches. c Profiles for $W=110 \mathrm{~mm}$ at the six Froude numbers shown in panel $\mathbf{d}$, which plots the avalanche dura- tion (time from maximum to minimum of the avalanche profile) and the characteristic time $t_{1 / 2}$. Blue (red) data represent the drum with $W=287 \mathrm{~mm}(17 \mathrm{~mm})$, and the gray crosses (circles) show corresponding direct measurements of $t_{A}$. e Mean scaled avalanche profiles at the six Froude numbers for $W=17 \mathrm{~mm}$ (color figure online) on and off: the kink occurs when the advancing front that mobilizes flow reaches the bottom of the sand surface and reflects into the retreating front that arrests motion.

The preceding observations suggest a physical picture of sand avalanche dynamics: in the wider drums, the two fronts fully traverse the granular surface. The triggering front takes time to start at the lower rotation rates, increasing the avalanche time and reducing the memory on the starting angle. When the arresting front returns to the top of the drum to switch off the avalanche, it partially sets the packing there, dictating when the next avalanche begins and correlating $\theta_{\text {stop }}$ with the following $\theta_{\text {start }}$. In the narrower drums, sidewall friction slows and weakens the arresting front so that the avalanche takes longer to terminate and the packing at the top of the drum is set by an earlier collapse, decorrelating $\theta_{\text {stop }}$ and the next $\theta_{\text {start }}$.

\subsection{Sand spectra}

A selection of power spectra for the sand are shown in Fig. 24; the frequency of the dominant peak is plotted against rotation rate in Fig. 25. Below transition, the enhanced periodicity of episodic avalanching is highlighted by the sharpness of the main spectral peak and the multiplicity of its harmonics. Above the transition to continuous flow, the spectra become broadly peaked and red. Strong additional peaks also appear at low frequency at the higher rotation speeds which are not connected to the motor contaminations. Direct observations of the drum indicate that these peaks correspond to coherent oscillations of the granular surface, in the manner of some sort of sloshing mode of the flowing layer. For example, at the higher speed in Fig. 24, $\Omega=0.4 \mathrm{rad} \mathrm{s}^{-1}$, a sloshing mode arises with a frequency of about $2.7 \Omega$.

Figure 25 illustrates the clear switch in the dominant spectral peak when the transition is hysteretic (compare the blue and red points). For the continuous flow data plotted in this figure sloshing modes have yet to appear and the spectral peak characterizes noisy flow fluctuations. The resulting characteristic frequency is clearly distinct from that for episodic avalanching, which once more reflects the typical avalanche spacing (fits of the form (8) again furnish a fair representation of the data; see Fig. 25). Evidently, the transition arises when the slumping and rolling frequencies are well matched, much as suggested previously in some qualitative prescriptions (e.g. [17,27]). 


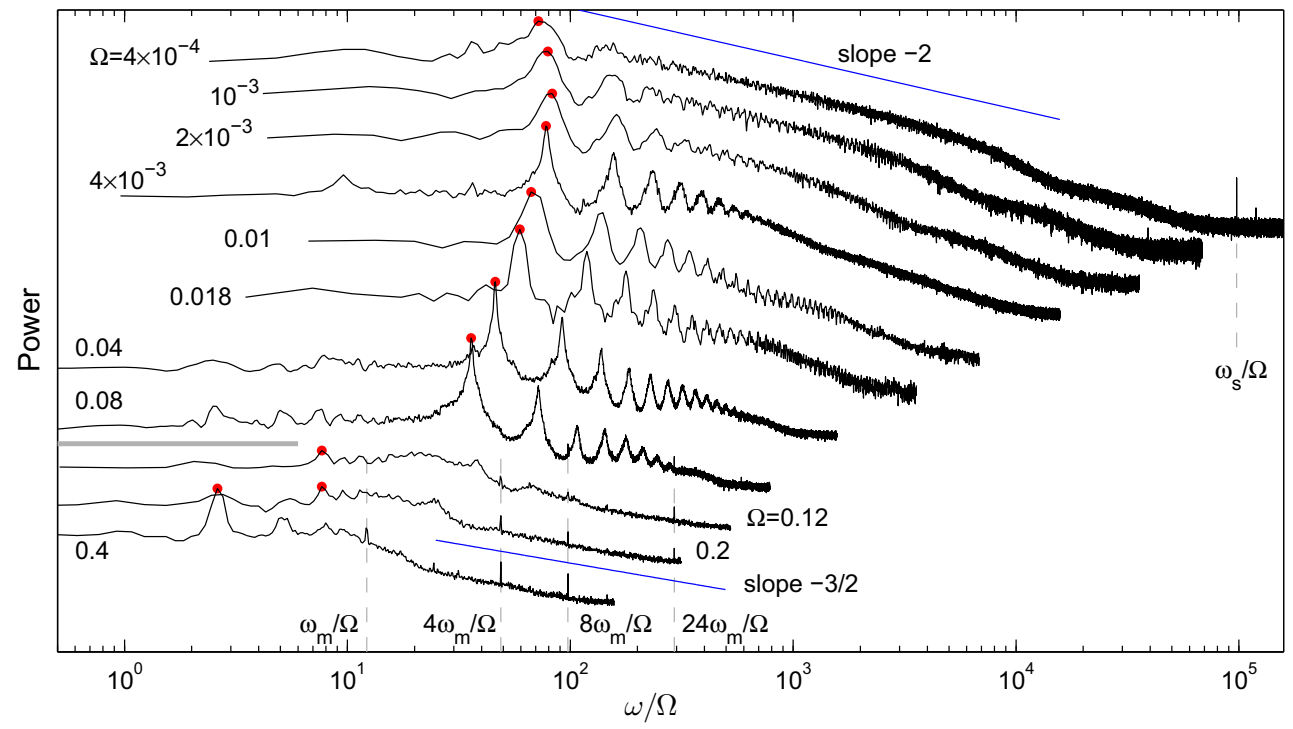

Fig. 24 Power spectra for sand in the drum with $(D, W)=$ $(287,110) \mathrm{mm}$, plotted against $\omega / \Omega$, for the rotation rates indicated (with both $\Omega$ and $\omega$ in $\mathrm{rad} / \mathrm{s}$ ). The spectra are offset for clarity and the dominant peaks are identified. Vertical dashed lines show the additional

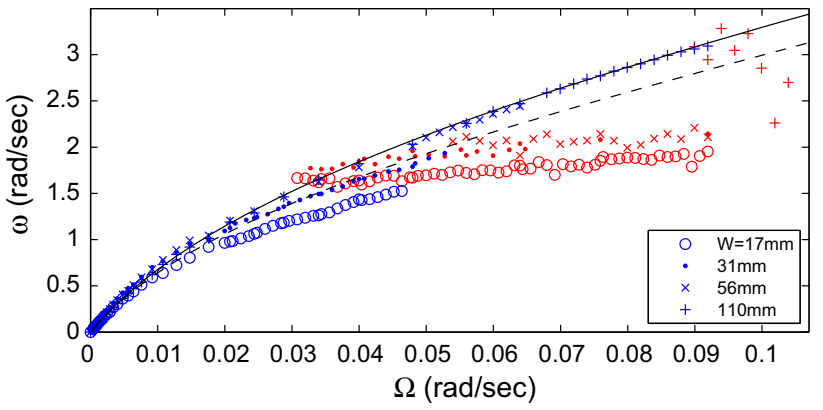

Fig. 25 The frequency of the dominant peak in the power spectra for sand in the big drum with the widths indicated. Blue (red) symbols indicate frequencies in the episodic avalanching (continuous flow) regime. The dashed line denotes the fit in (8) to the $W=110 \mathrm{~mm}$ data, using a fit to the observed $t_{A}$ (Fig. 40) and the mean avalanche amplitude over the episodic regime $\langle\Delta \theta\rangle \approx 4^{\circ}$; the solid line uses least squares with (8), including $\Delta \theta, t_{0}$ and $\Gamma$ as fitting parameters (color figure online)

\section{Conclusions}

In this paper we have reported an experimental survey of episodic avalanching and continuous flow in a granular drum. This device is a classical arrangement to study granular dynamics, yet a detailed investigation of the two regimes and the transition between them has not previously been presented. We considered a variety of different granular media (glass spheres with a range of diameters and a sand) and drums with different diameters and widths. Our main advance was to catalog the dynamics over lengthy sweeps in rotation rate, afforded by an efficient data acquisition system. frequencies, $\omega_{s}=8000 \omega_{m}$ and $\omega_{m}$, and some of their harmonics. The transition is indicated by the gray horizontal line. The inclined dashed lines indicates slopes of -2 and $-3 / 2$

Sweeps could be conducted for days or even weeks, allowing us to collect relatively clean statistics of episodic avalanching or the vagaries of continuous flow. From the experiments we have characterized the properties of the two regimes and the intervening transition, which sets the stage for modeling efforts to match the observations and test theories.

For most of our spheres and drums, the transition from episodic avalanching to continuous flow takes the form of a gradual switch in dynamical behavior wherein the two phases are blended in varying degrees. As the particle radius becomes small, the blend becomes refined into an alternation between clearly defined phases of continuous flow or episodic avalanching (cf. [12]). For sand, the transition is again intermittent in either the biggest or smallest drums, but in all others a hysteretic transition takes place [32]. Overall, the different forms of the transition are suggestive of a system in which there are two possible states and which is perturbed by differing degrees of noise. The transition is a smooth blend in behavior for higher noise levels, hysteretic for weak noise, and intermittent in between. There is little sign that the continuous flow state disappears in some kind of a deterministic bifurcation at low rotation rates, or that episodic avalanching terminates at higher rotation rates in another bifurcation. In other words, the transition explored here is noisier, but follows the general scenario outlined in [12].

The results for glass spheres suggest that the transition is largely independent of drum width and arises roughly for $0.3<\operatorname{Fr}(D / d)<0.5$, in terms of the Froude number, $\mathrm{Fr}=\Omega \sqrt{D / g}$. This criterion is consistent with previously 
reported results for spheres [3,12], but does not work for sand which displays a more complicated dependence on the drum geometry. The criterion is different to a number of existing predictors of transition based mostly on heuristic arguments, although it is similar to one proposed by Liu et al. [24] for angular particles such as sand, but not glass spheres. In any event it is hard to see how to reconcile the heuristic arguments underlying these other predictors with the nature of the transition as they take no explicit account of effect of dynamical noise. We also find little support for a suggestion [19] that the transition is connected to the flow-depth-surface-angle relation for sheet flow to cease on an inclined plane (see Appendix B).

Although we have resisted providing any detailed theoretical models to complement our experiments, these are certainly possible. Indeed, we conducted simulations with the Discrete Element Method in tandem with the experiments, and which helped guide some of our scalings and fits of the data. A brief discussion of a model for a relatively narrow drum based on the $m u(I)$ law is provided in Appendix B. One can also build cruder ODE models (e.g. [4]). To capture the experimental observations, stochastic forcing is essential in these models. Moreover, two types of noise are needed: fluctuations in packing are required to furnish a random starting angle for an avalanche, and dynamical noise during flow is needed to recover a random stopping angle. With both types of noise suitably incorporated, models can be designed that show some qualitative agreement with the observations. However, many of the finer details (such as the distributions and correlations of the starting and stopping angles) are likely to be awry without additional empirical input.

A persistent ageing effect plagued our efforts to generate reproducible results. We eliminated this feature by suitably maturing particles in high-speed burn-in experiments, and by restricting our use of glass spheres with smaller diameters. Polydispersivity may constitute another intrinsic problem, with segregation potentially also leading to long-time evolution. Overall, the surface angle during both episodic avalanching or continuous flow is sensitive to ageing effects and drum or particle geometry, and is likely to be significantly affected by external noise in less controlled situations. One should exercise caution in using such a statistic to characterize granular dynamics (cf. [21]); other, more robust measures offer greater diagnostic value.

Beyond the rotating drum, one may wonder what kinds of intermittent motions occur in other flow configurations. Episodic avalanches also arise in heap flows and sandpiles fed at low flux (e.g. [2,19,22]), and similar intermittent motions occur underneath plates dragged over granular layers [28]. In sheet flow down an incline (e.g. [13]) or for bulldozed sandpiles [35], however, episodic avalanching does not occur at low flow rates. For the inclined plane, fluctuations during continuous flow trigger the arrest of flow, but once grains stop noise cannot drive the system back into motion, precluding any recurring slumping state. For the bulldozer, there is again a continuous flow state, but the driving appears to preclude any locked-up arrangement like rigid rotation. Stochastic fluctuations can then only agitate the system about the continuous flow state.

Acknowledgements We thank Greg Wagner and Quentin Debray for assistance and discussions.

\section{Compliance with ethical standards}

Conflict of interest The authors declare no conflict of interest.

Open Access This article is distributed under the terms of the Creative Commons Attribution 4.0 International License (http://creativecomm ons.org/licenses/by/4.0/), which permits unrestricted use, distribution, and reproduction in any medium, provided you give appropriate credit to the original author(s) and the source, provide a link to the Creative Commons license, and indicate if changes were made.

\section{Appendix}

\section{A Experimental details}

\section{A.1 The motor for the bigger drum}

The larger cylinder was driven by a high performance stepper motor (a Parker Compumotor iBE342H), which could rotate stably very low speeds. Given that episodic avalanching could be sensitive to variations in motor speed we estimated the precision of this device by monitoring time series of the angular position of the shaft. In its velocity-controlled mode, the motor has settings to improve speed stability; using suitable choices for these (in the Parker nomenclature, we used $\mathrm{KI}=0, \mathrm{KP}=10$ and $\mathrm{KD}=1000$, for the integral, proportionality and damping constants, respectively), the angular position error was always less than 0.06 degrees over the duration of each experiment. However, velocity fluctuations do occur during each run as the motor adjusts to maintain the angular position. The inertia and compliance of the drum apparatus likely lessen these variations, but our best estimates suggested that rotation rate errors of order $5 \times 10^{-5}$ $\mathrm{rad} / \mathrm{s}$ could occur over intervals of several seconds. These errors are somewhat lower than the smallest rotation rates used, but the associated accelerations may contribute to the ambient agitation of the apparatus and trigger avalanches.

\section{A.2 Image processing}

The camera was orientated so that the granular surface was roughly horizontal, with vertical plumb lines included in the field of view to determine the precise orientation. Images 

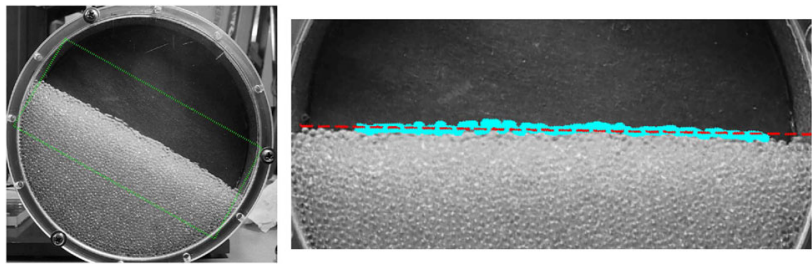

Fig. 26 An image taken of the $2 \mathrm{~mm}$ spheres in the $137 \mathrm{~mm}$ diameter drum $(W=17 \mathrm{~mm})$, together with the extraction of the surface profile and its linear fit

were processed in real time, mostly at $20 \mathrm{~Hz}$, with the left and right edges of the drum image clipped by $2 \mathrm{~cm}$ to assist with the fitting of the slope of the granular surface. The surface was first identified by finding the highest pixel in each vertical raster of the image in which the intensity exceeded a threshold set to be halfway between the raster's minimum and maximum. The best fit straight line to the identified pixels was found and all points lying at least ten pixels from the fit marked as outliers and discarded. The line was fit a second time and any new outliers again discarded. Repeating the procedure one more time furnished the slope angle $\theta(t)$; an example is shown in Fig. 26. The procedure does not therefore fit the entire surface profile, and is misleading when the drum is rotating relatively quickly and the characteristic S-shape develops. As diagnostics of the goodness of fit, we recorded the mean squared error and the fraction of discarded points (which was typically less than 5\%). We also constructed average images and the differences between successive images in order to furnish mean surface profiles and examine the apparent flowing layer depth.

Although the surface is relatively flat for most of our experiments, the profile becomes nonlinear as the drum is rotated more quickly and its width is reduced. Figure 27 displays mean surface profiles for the fastest drums. The characteristic S-shape develops at these speeds, becoming most prominent for the biggest diameter, narrowest drums and the smallest particles. Note that the surface profiles in the two widest drums ( $W=110$ and $205 \mathrm{~mm}$ ) are practically identical, indicating that the dynamics is insensitive to the width for these cases, yet the S-shape still develops.

For each time series of $\theta(t)$, the initial $10 \mathrm{~s}$ were clipped to remove transients. A median filter over a width of 5 samples was then applied to reduce noise. The starting and stopping angles, $\theta_{\text {start }}$ and $\theta_{\text {stop }}$, were identified by first calculating the standard deviation $\sigma$ of $\theta(t)$. For a time series with a periodic triangular waveform of peak-to-peak amplitude $\Delta \theta$, the distribution of $\theta$ is uniform and $\sigma=\Delta \theta / \sqrt{12}$. We therefore chose an angle threshold $\delta_{\theta}=\frac{1}{5} \Theta / \sqrt{12}$ designed to detect avalanches of amplitude down to about $1 / 5$ of the average. If the avalanche time is much less that the time for solidbody rotation, this corresponds to a time interval between avalanches of $\delta_{t}=\delta_{\theta} / \omega$. The moving maximum over $\theta$ corresponding to this time interval is then found and the start of each avalanche identified as the times where $\theta$ equals this maximum. We then find all the minimum values between each pair of maxima to locate the end of each avalanche. Finally the candidate list of avalanches is searched and any that are smaller than $\delta_{\theta}$ are removed. The procedure is robust and copes well with noise, but clips the smallest avalanches.

Given the time series $\theta\left(t_{i}\right)=\theta_{i}$ ( $t_{i}$ being the $i^{t h}$ sampling time), we define the distance skewness of the rate of change $\dot{\theta}_{i}=\left(\theta_{i+k}-\theta_{i}\right) /\left(t_{i+k}-t_{i}\right)$, by

$$
D_{\text {skew }}\left(t_{k}\right)=1-\frac{\sum_{i, j}\left|\dot{\theta}_{i}-\dot{\theta}_{j}\right|}{\sum_{i, j}\left|\dot{\theta}_{i}+\dot{\theta}_{j}\right|},
$$

for a prescribed delay time $t_{k}$. For each time series we optimised the choice for $t_{k}$ by increasing $k$ until $D_{\text {skew }}$ reached a maximum, and then adopted the maximal value.
Fig. 27 a Surface profiles for the drum with $D=289 \mathrm{~mm}$ and varying width, filled with $2 \mathrm{~mm}$ (red, vertically offset) and $3 \mathrm{~mm}$ (blue) spheres. The insets show the same data, but for the drum with $D=137 \mathrm{~mm}$. b Surface profiles for particles with diameter $d=1,2$ and $3 \mathrm{~mm}$, in the drum with

$(D, W)=(287,110) \mathrm{mm}$, for $\Omega=0.44,0.66$ and $0.88 \mathrm{rad} \mathrm{s}^{-1}$ (color figure online) (a) surface profiles varying $\mathrm{W}, \Omega=0.44 \mathrm{rad} / \mathrm{s}$

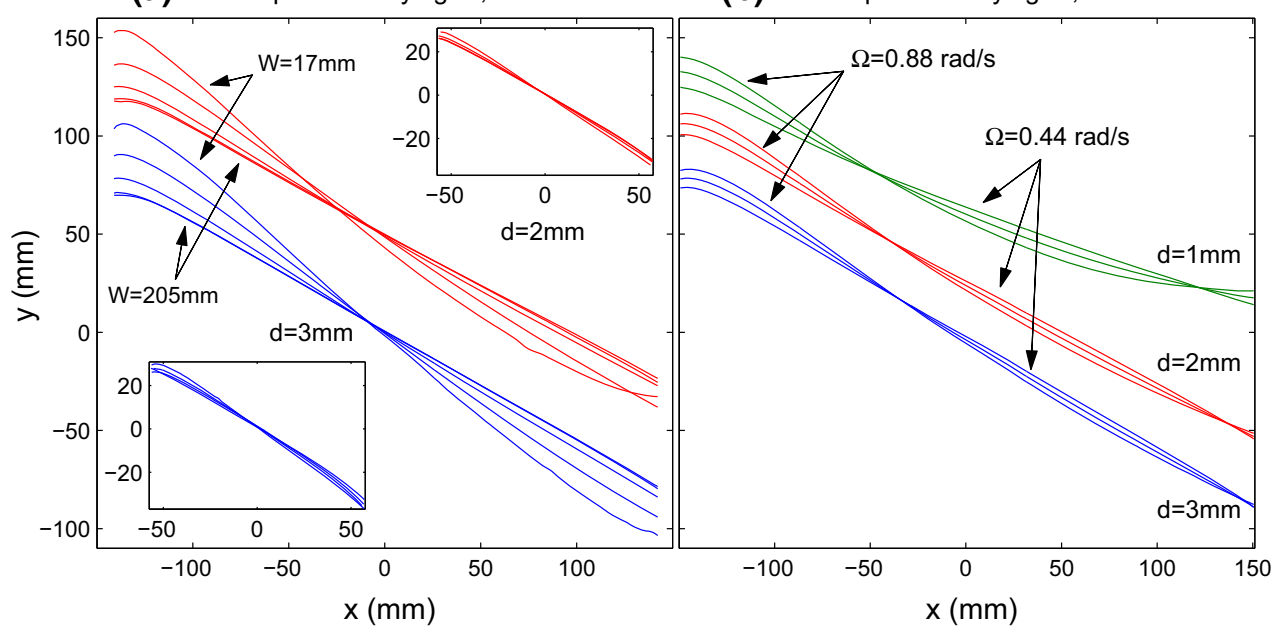




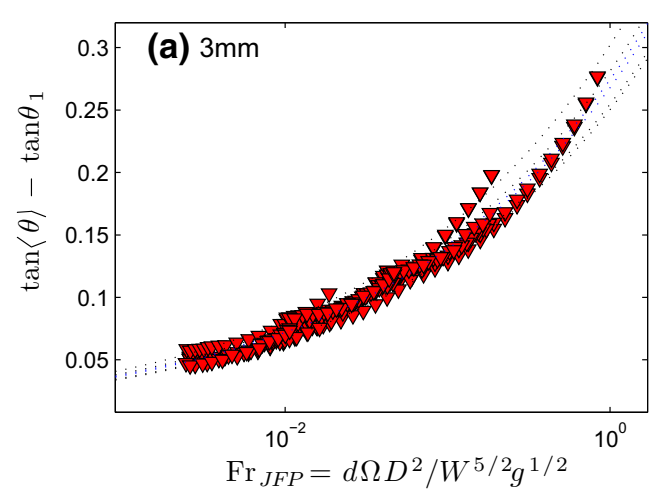

Fig. 28 a Mean angle data in continuous flow $\left(D_{\text {skew }}<0.01\right)$ for $3 \mathrm{~mm}$ glass spheres in the narrower drums, $3 W<D$, plotting $\tan \langle\theta\rangle-\tan \theta_{1}$ against $\operatorname{Fr}_{J F P}=d \Omega D^{2} / \sqrt{g W^{5}}$, where $\tan \theta_{1}$ is the intercept deter-

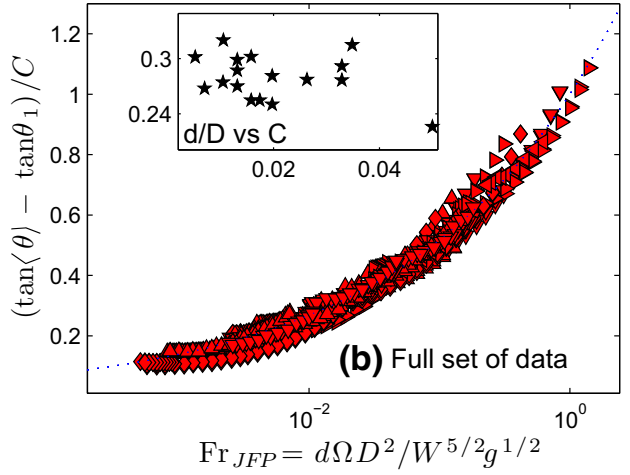

mined from a linear fit of $\tan \langle\theta\rangle$ to $\mathrm{Fr}_{J F P}^{2 / 7}$, (dotted lines). b Mean angles for glass spheres, plotting $\left(\tan \langle\theta\rangle-\tan \theta_{1}\right) / C$ against $\operatorname{Fr}_{J F P}$, where $C$ is the slope of the linear fits. The symbol convention follows the legend of Fig. 6. The inset plots $C$ against $d / D$
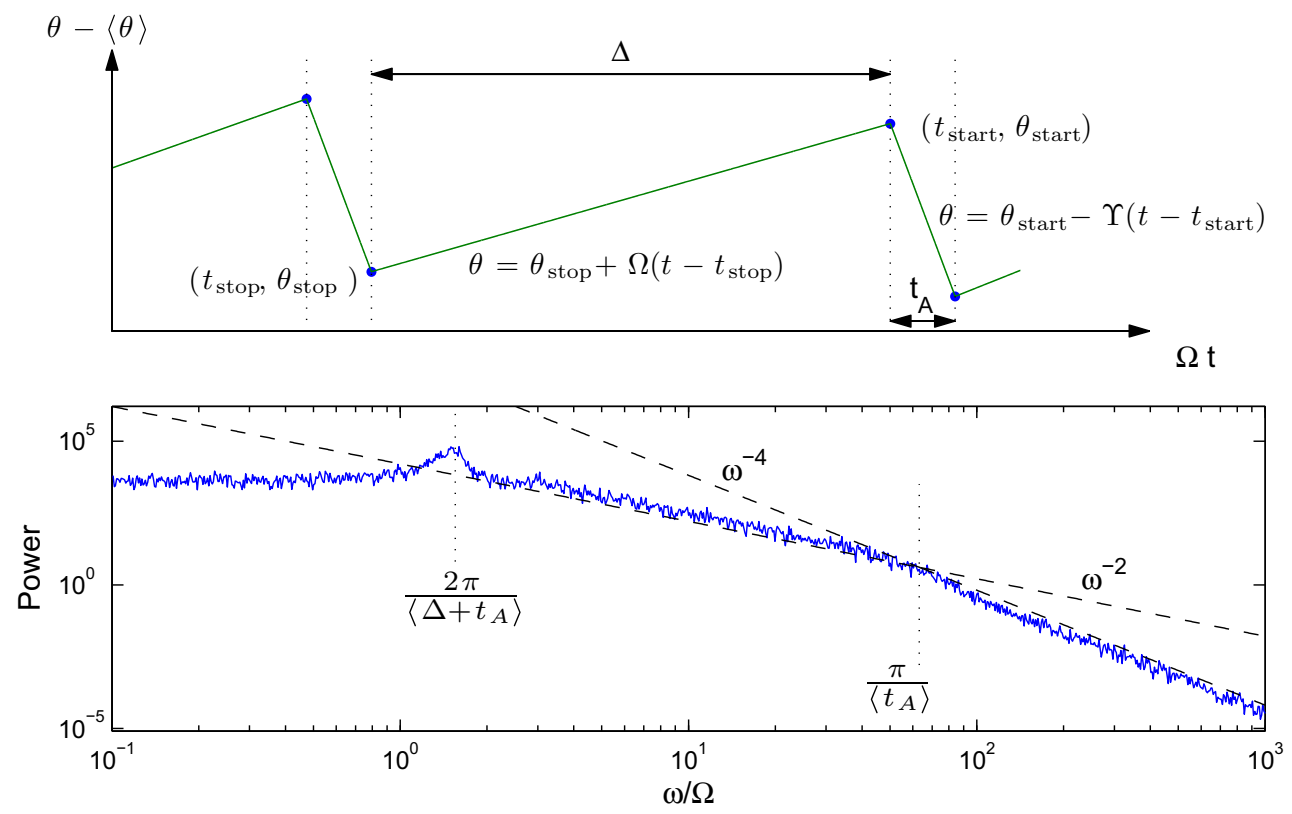

Fig. 29 a Synthetic time series of the surface angle constructed from random variables representing the starting and stopping angles, $\theta_{\text {start }}$ and $\theta_{\text {stop }}$, and the duration of the avalanches, $t_{A}$. b Average power spectrum from ten realizations of the synthetic time series, based on
$N=2000$ avalanches. $\theta_{\text {start }}$ and $\theta_{\text {stop }}$ are chosen from Gaussian distributions with means separated by $\langle\Delta \theta\rangle=4^{\circ}$ and standard deviations of 0.5 , and $\Omega t_{A}$ selected from a Gaussian distribution with mean 0.05 and standard deviation 0.0125

Jop et al., one can compute the flow depth and flux if

$\mu(I)=\frac{I_{0} \mu_{1}+I \mu_{2}}{I_{0}+I}$

where $\mu_{1}, \mu_{2}$ and $I_{0}$ are material parameters. The flowing layer depth is

$\delta=\frac{W}{\mu_{w}}\left(\tan \langle\theta\rangle-\mu_{1}\right)$, 

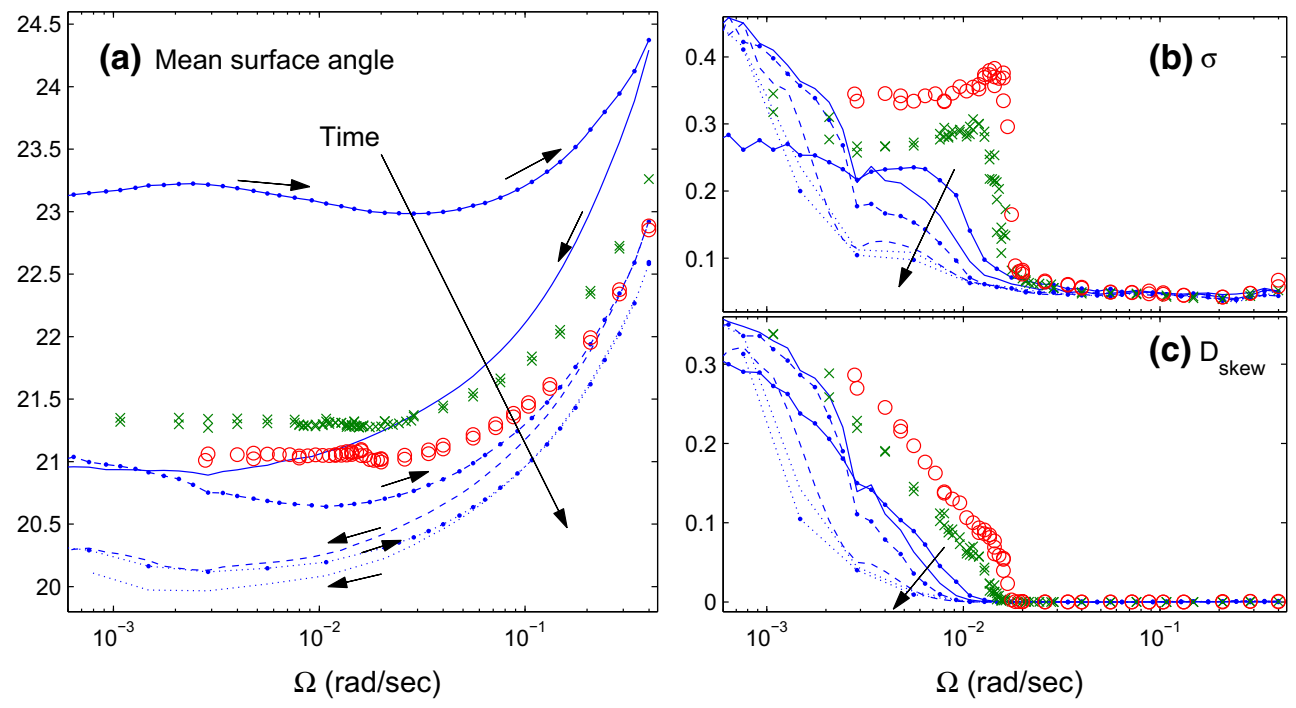

Fig. 30 Repeated sweeps conducted with a fresh sample of $1 \mathrm{~mm}$ spheres in a drum with $(D, W)=(287,110) \mathrm{mm}$, showing a the secular decrease of the mean surface angle $\langle\theta(t)\rangle$, $\mathbf{b}$ the standard deviation $\sigma$, and $\mathbf{c}$ the distance skewness $D_{\text {skew }}$. The repeated sweeps are shown by lines (in the up/down repeated sequence dots/solid, solid, dots/dashed, dashed, dots/dotted, dotted); the angle change is over twice that for $d=3 \mathrm{~mm}$ shown in Fig. 1 of the main text. The circles and crosses

show two up-down sweeps for spheres that were matured as follows: the spheres were aged in a first burn-in run, then removed from the drum and washed, and finally aged in a burn-in run (see Fig. 31). The slumping-to-rolling transition then becomes more robust but the results are still not especially reproducible, with significant discrepancies arising between the two batches of similarly prepared particles

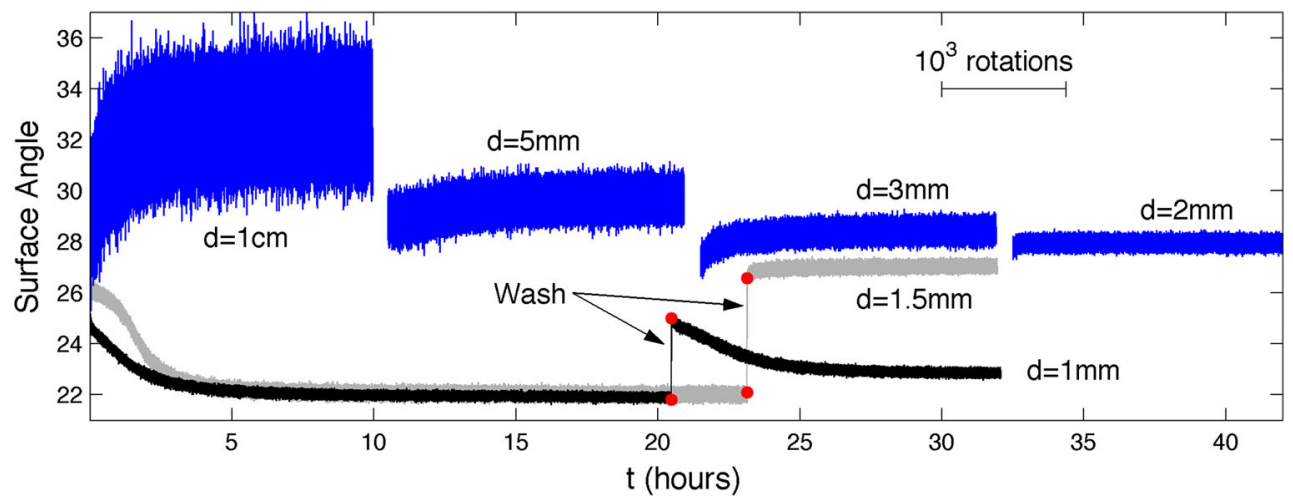

Fig. 31 Burn-in runs, showing the drift of the surface angle at fixed speed $(0.4 \mathrm{rad} / \mathrm{s})$ for the glass spheres indicated in the $(D, W)=$ $(287,110) \mathrm{mm}$ drum. The upward shift of the mean angle is evident for the spheres with diameter $d>2 \mathrm{~mm}$. For $d=2 \mathrm{~mm}$, there is barely any drift; the 1.5 and $1 \mathrm{~mm}$ particles both drift downward. For 1.5 and

where $\mu_{w}$ is the coefficient of sliding friction over the walls, and the net flux is

$$
\begin{aligned}
q=2 I_{0} & \frac{\sqrt{g \cos \langle\theta\rangle}}{5 d}\left[W \frac{\left(\mu_{2}-\mu_{1}\right)}{\mu_{w}}\right]^{5 / 2} F\left(\frac{\delta \mu_{w} W^{-1}}{\mu_{2}-\mu_{1}}\right) \\
F(X)= & 5(1-X)\left[\sqrt{1-X} \sin ^{-1} \sqrt{X}-\sqrt{X}\right] \\
& +\frac{5}{3} X^{3 / 2}-X^{5 / 2}
\end{aligned}
$$

$1 \mathrm{~mm}$ spheres, the material is washed after the first burn, and then aged a second time. For the $1.5 \mathrm{~mm}$, the washing of the particles reverses the sense of the drift during the second burn-in, with the mean angle then increasing. To display the data without any overlap, some of the time traces are shifted horizontally

For the drum, $q=\frac{1}{8} \Omega D^{2}$, and so (11) furnishes an algebraic equation for $\theta$. The full algebraic form of (12) is a little unwieldy and its value is not so different from the small$X$ approximation, $F \approx \frac{2}{7} X^{7 / 2}$. If we adopt the latter as an approximation, we find

$\tan \theta=\mu_{1}+\left(\frac{\mu_{w}}{W}\right)^{5 / 7}\left[\frac{35 d R^{2} \Omega\left(\mu_{2}-\mu_{1}\right)}{8 I_{0} \sqrt{g \cos \langle\theta\rangle}}\right]^{2 / 7}$.

This relates $\tan \langle\theta\rangle-\mu_{1}$ to $\operatorname{Fr}_{J F P}$, as plotted in Fig. 28, and is equivalent to the scaling of heap-flow data by Jop et al. For the 


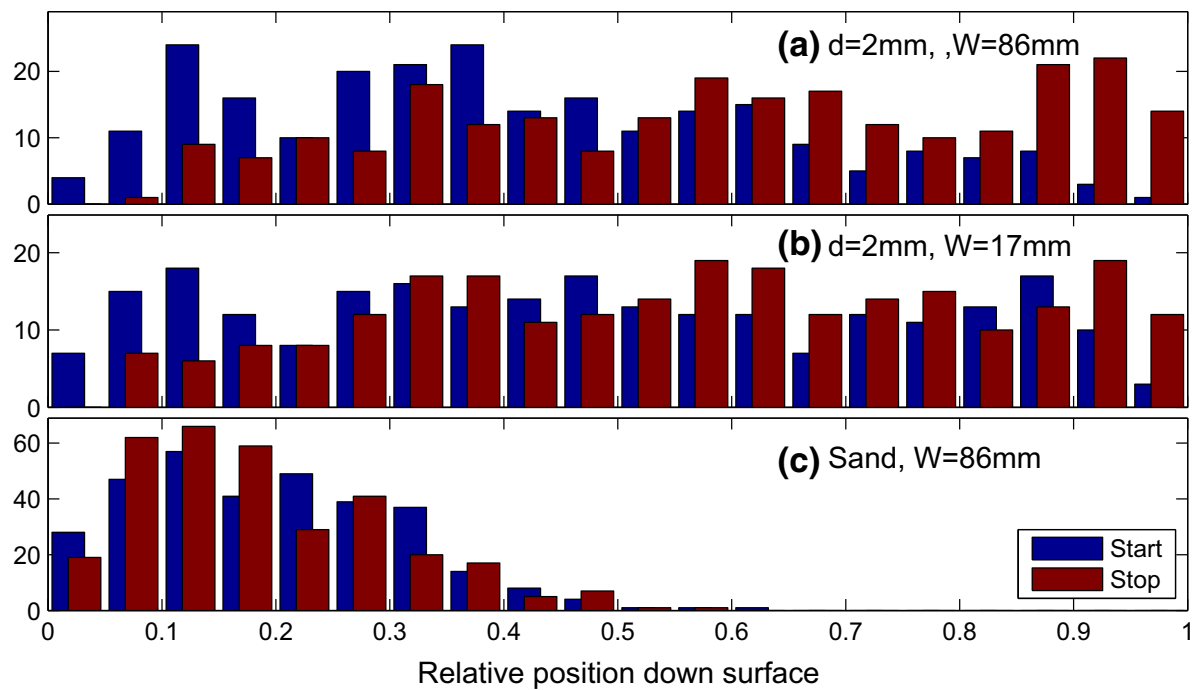

Fig. 32 Histograms of the relative starting and stopping positions of avalanches down the granular surface (defined so that 0 refers to the top of the inclined surface and 1 to the bottom in the image taken by the video camera) for $d=2 \mathrm{~mm}$ glass spheres and sand in the drum with
$D=137 \mathrm{~mm}$ and the widths indicated. The position is determined by locating the pixels of the first or last coherent motions away from rigid rotation (discarding individual grain motion), or by taking the average of such positions if multiple locations are indistinguishable
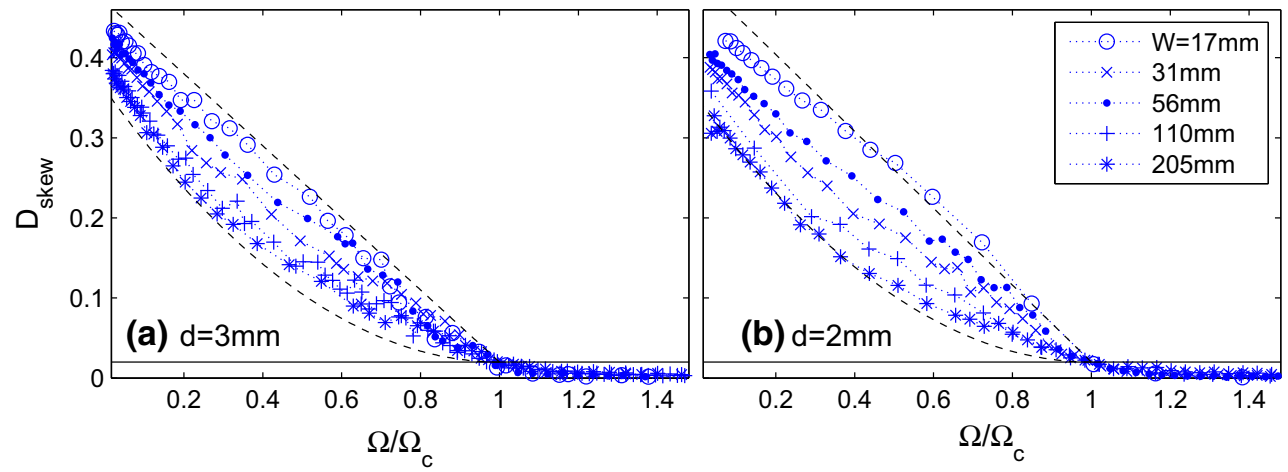

Fig. $33 D_{\text {skew }}$ plotted against $\Omega / \Omega_{c}$ for $\mathbf{a} d=3 \mathrm{~mm}$ and $\mathbf{b} d=2 \mathrm{~mm}$ spheres from the larger drum $(D=289 \mathrm{~mm})$, with the different symbols corresponding to the widths indicated. As in Fig. 6, $\Omega_{c}$ is the interpolated rotation rate for which $D_{\text {skew }}=D_{\text {crit }}=0.02$ (this threshold is also indicated). The diagnostic increases more sharply in the narrower drums than the wider ones as episodic avalanching commences; the dashed lines show the fits, $D_{\text {skew }} \propto\left(1-\Omega / \Omega_{c}\right)$ and $D_{\text {skew }} \propto\left(1-\Omega / \Omega_{c}\right)^{3 / 2}$. Note that the observed $D_{\text {skew }}$ do not abruptly reduce to zero but trail off gradually as one passes through $\Omega=\Omega_{c}$, much as in an imperfect bifurcation

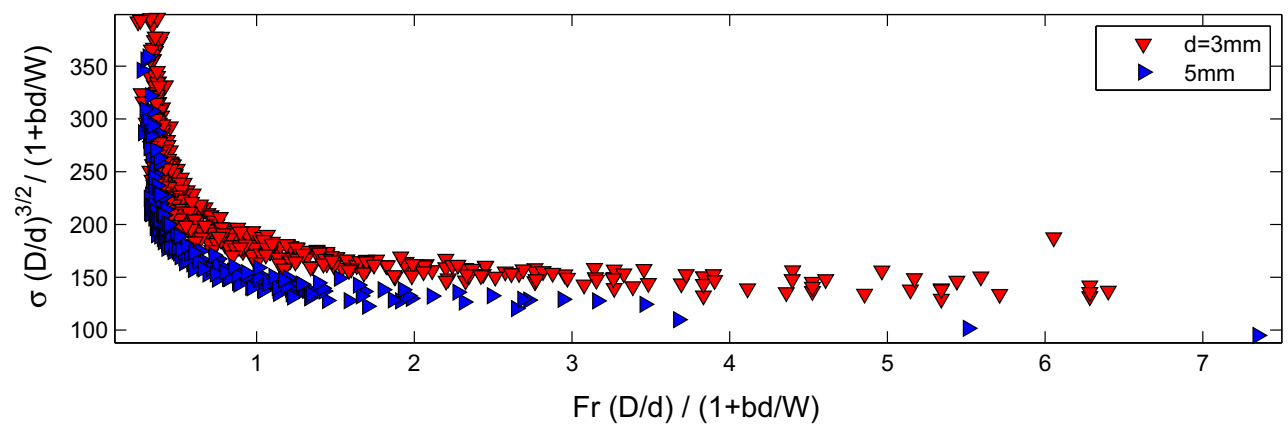

Fig. 34 Collapse of the standard deviation data in the rolling regime $\left(D_{\text {skew }}<0.01\right)$ against Froude number, both scaled as indicated, for $d=3$ sand $5 \mathrm{~mm}$. For $\sigma$ scaled by $\left.(D / d)^{3 / 2} /(1+3 d / 2 W)\right)$ i.e. $\left.b=1.5\right)$, there is a fair degree of collapse for these two sphere diameters. However, the scaling does not collapse the two sets of data, and works less well for other values of $d$. The figure serves mainly to illustrate how the fluctuations in the rolling regime sharply increase as $\Omega \rightarrow 0$, suggesting that the continuous flow state inevitably becomes disrupted at low flow rates by noise, triggering the transition to episodic avalanching 

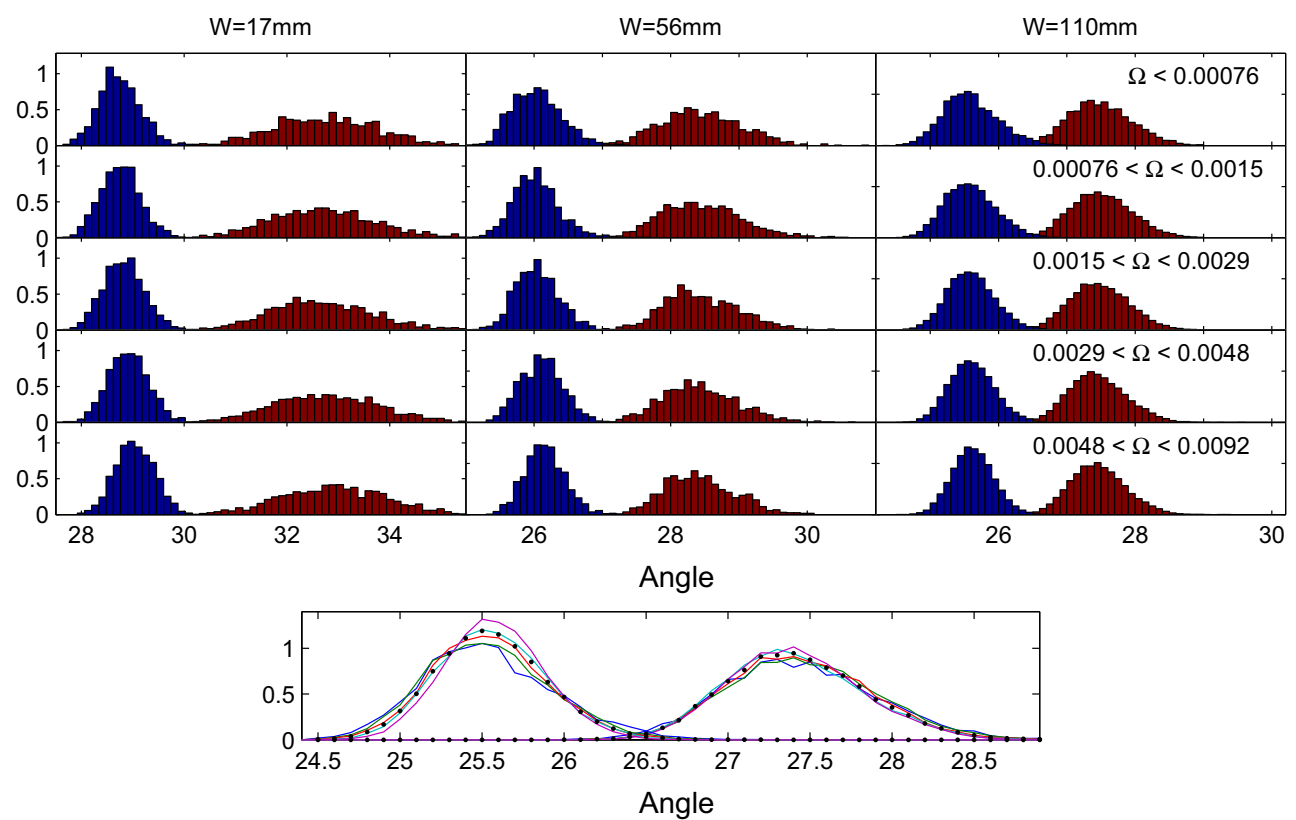

Fig. 35 Histograms of $\theta_{\text {start }}$ and $\theta_{\text {stop }}$ for $d=3 \mathrm{~mm}$ spheres in the drum with $D=287 \mathrm{~mm}$ and the widths and rotation rates (in rad/s) indicated. The final panel compares the distributions for $(D, W)=(287,110) \mathrm{mm}$; the dots show the average over the slumping regime

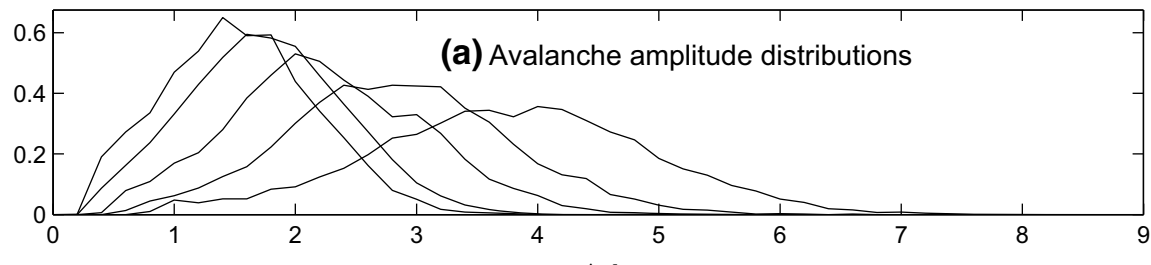

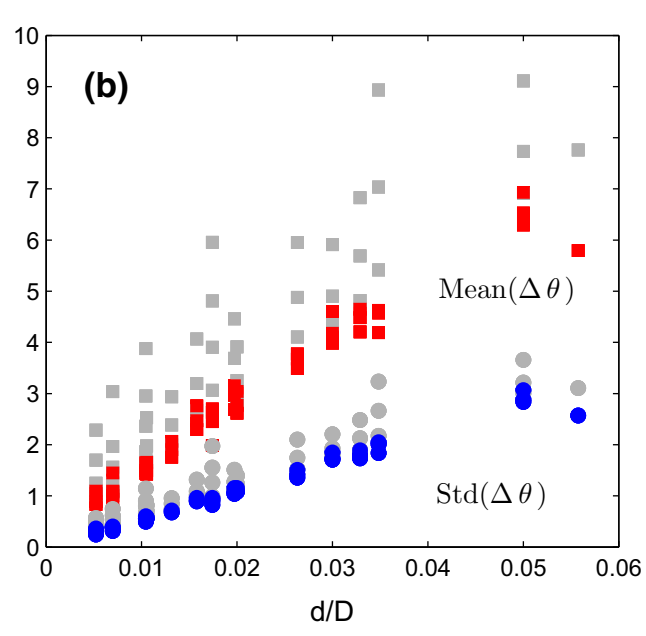

Fig. 36 a Avalanche amplitude distributions for $(d, D)=(3,287) \mathrm{mm}$ and $W=17,31,56,110$ amd $205 \mathrm{~mm}$ (as shown in Fig. 9). b The mean and standard deviation of the distribution as a function of $d / D$; gray points show the raw data, the darker (red and blue) points after the drum width dependence is removed using a linear fit with $d / W$. c, $\mathbf{d}$ The

lower rotation rates, the data bend away from the prediction, reflecting the impending transition to episodic avalanching. At the higher rotation speeds, the data again bend away from the data, possibly due to inertia (the approximation of $F(X)$
$\Delta \theta$

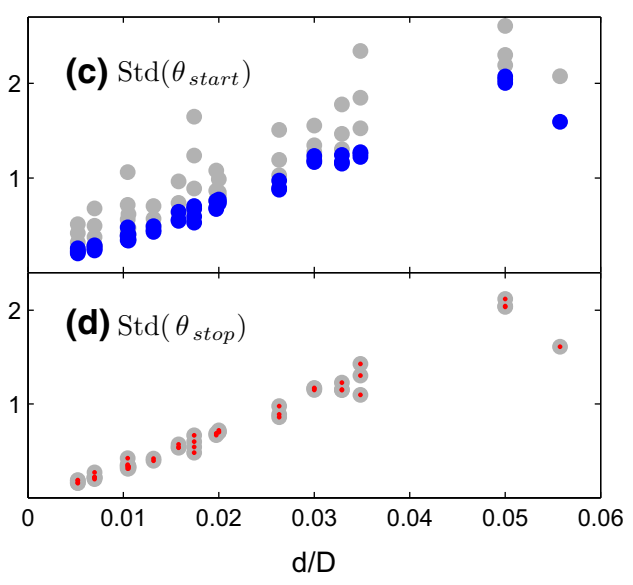

standard deviation of the $\theta_{\text {start }}$ and $\theta_{\text {stop }}$ distributions. $\operatorname{Std}\left(\theta_{\text {stop }}\right)$ shows no significant dependence on drum width; the drum width dependence of $\operatorname{Std}(\Delta \theta)$ therefore originates purely from that of $\theta_{\text {start }}$ (color figure online)

is not responsible). The limiting friction $\mu_{1}$ and the factor $\mu_{w}^{5 / 7}\left[35\left(\mu_{2}-\mu_{1}\right) / 8 I_{0} \sqrt{\cos \langle\theta\rangle}\right]^{2 / 7}$ provide interpretations of the intercept $\tan \theta_{1}$ and slope $C$ of the fits in Fig. 28. 


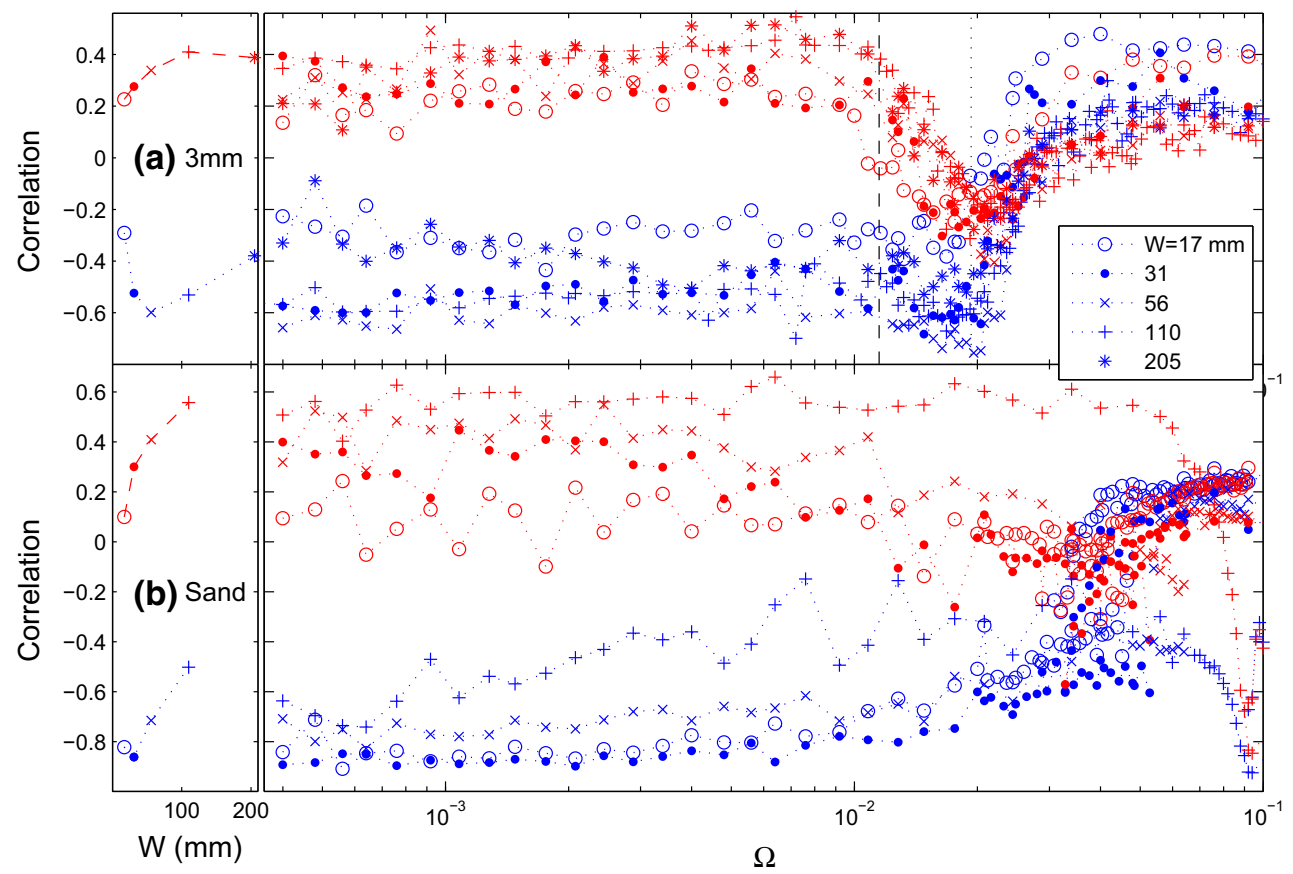

Fig. 37 Correlation coefficients between $\theta_{\text {start }}$ and the following $\theta_{\text {stop }}$ (blue) and the previous $\theta_{\text {stop }}$ (red) plotted against $\Omega$ (in $\mathrm{rad} / \mathrm{s}$ ) for a $3 \mathrm{~mm}$ spheres and $\mathbf{b}$ sand in the big drum. Each data point is averaged over all experiments at the same rotation rate; for the hysteretic sand data, the averages are taken only over experiments with the same phase.
The symbols refer to drum width, as indicated. The panels on the left show averages for $\Omega<0.01 \mathrm{rad} \mathrm{s}^{-1}$, plotted against $W$, in a, the vertical dotted and dashed lines indicate the upper and lower edges of the transition region, ignoring the weak width-dependence (color figure online)

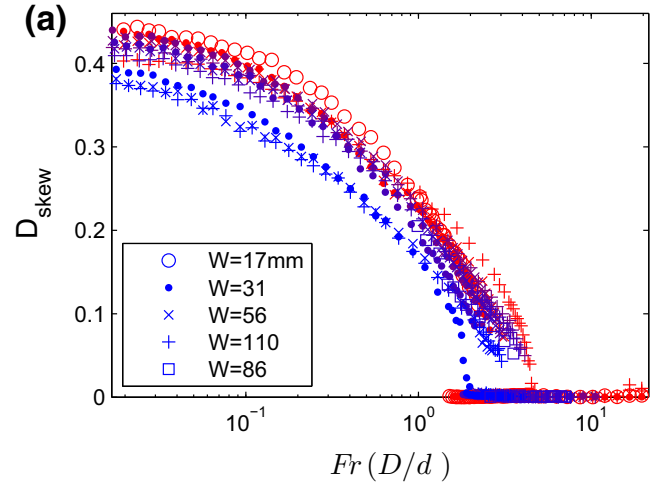

Fig. 38 a $D_{\text {skew }}$ and $\mathbf{b} \sigma$ for sand, scaled as indicated and plotted against $\operatorname{Fr}(D / d)$, which collapses the data close to a common curve. Each data point is an average over all experiments with the same $(\Omega, D, W)$ and phase (episodic avalanching or continuous flow), and the points are color coded according to drum diameter (red for $D=100 \mathrm{~mm}$ and blue

Jop et al. suggest that episodic avalanching begins when the flow-depth-flux relation intersects the $h_{\text {stop }}(\langle\theta\rangle)$ curve for sheet flow down an inclined plane (i.e. the depthangle relation holding when flow ceases on reducing the incline). Because we have not measured $h_{\text {stop }}$-curves for our spheres, we cannot directly examine this supposition. However, near the onset of flow, previous results suggest that $h_{\text {stop }} \propto d\left(\tan \langle\theta\rangle-\mu_{1}\right)^{-m}$, with $m \approx 1$ [31]. Thus,

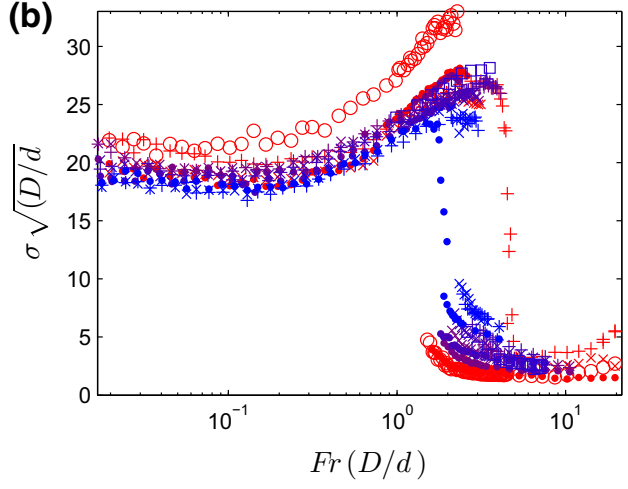

for $D=287 \mathrm{~mm}$ ). The plot conveys the impression that statistics of episodic avalanching are largely independent of $W$, but the transition to continuous flow takes place at a width-dependent Froude number (color figure online)

setting $\delta \sim d /\left(\tan \langle\theta\rangle-\mu_{1}\right)^{m}$, or $\delta \sim W(d / W)^{1 /(m+1)}$, suggests that the critical Froude number for transition scales as $\mathrm{Fr} \sim(W d)^{\frac{1}{2}(5-2 m) /(m+1)} / D^{3 / 2}$, in disagreement with (2).

For the wider drum, no equivalent theory exists to predict the depth of the flowing layer or flux from $m u(I)$. Nevertheless, assuming that the inertia number plays a key role, we 
(a) Avalanche amplitude distributions for $\mathrm{W}=110 \mathrm{~mm}$
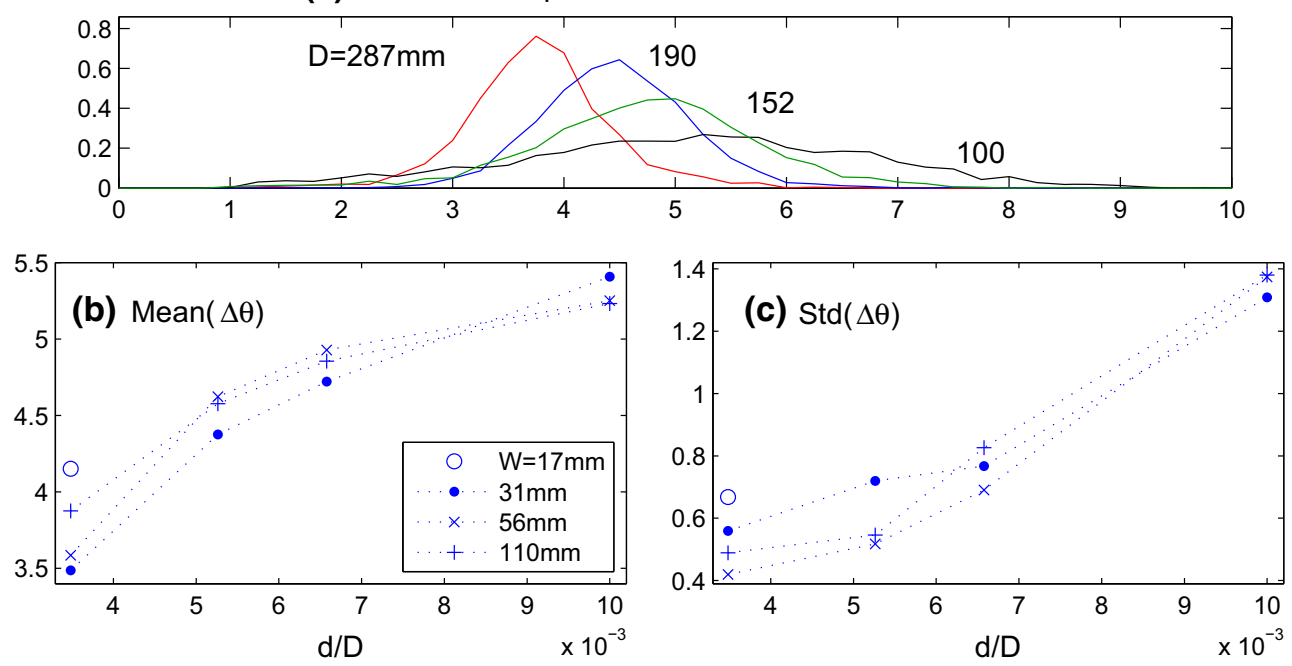

Fig. 39 a Avalanche amplitude distributions for sand in the drum with varying $D$ and $W=110 \mathrm{~mm}$. The mean and standard deviation of these distributions are plotted in $\mathbf{b}$ and $\mathbf{c}$, respectively. The distributions, means and standard deviations show no clear trend with drum width, unlike the glass spheres (cf. Fig. 36)

may dimensionally estimate an average,

$I \sim \frac{q d}{\delta^{2} \sqrt{g \delta}} \equiv \frac{d D^{2} \Omega}{8 \delta^{2} \sqrt{g \delta}}, \quad$ or $\quad \frac{\delta}{d} \sim\left(\frac{\mathrm{Fr}}{I}\right)^{2 / 5}\left(\frac{D}{d}\right)^{3 / 5}$

If the average $I$ is roughly constant, this estimate is not far from the previously reported scaling $(\delta / d) \sim \sqrt{\operatorname{Fr}}(D / d)^{3 / 4}$ [14]. The fit of $\langle\theta\rangle$ in (4) suggests that $C$ depends weakly on particle radius in relatively wide drums. In this case, Jop et al.'s strategy for locating transition furnishes a scaling, $\operatorname{Fr} \sim(d / D)^{3 /(2+5 m)}$, which again compares poorly with (2) for $\alpha \approx 1.1$ and $\beta d / W \rightarrow 0$.

We conclude that the mean angle scalings suggested by the thin-slot $m u(I)$ model are consistent with the drum data to a similar degree that Jop et al. account for their heap flow results (although, as emphasized by Fig. 8, our data is largely only linear over the experimental range). Our observed transition, however, does not appear to coincide with a criterion based on $h_{\text {stop }}(\langle\theta\rangle)$.

\section{Synthetic avalanche spectra}

We construct synthetic time series of the surface angle as illustrated in Fig. 29a: we randomly choose a sequence of values for the starting and stopping angles and the avalanche durations from Gaussian distributions. The signal is then built by adopting a linear rise at the rotation rate between a given stopping angle and the following starting angle, then inserting a linear collapse of duration $t_{A}$ to the next stopping angle. The time series for a realization of $N$ avalanches can be used to compute spectra, as illustrated in Fig. 29b, either by

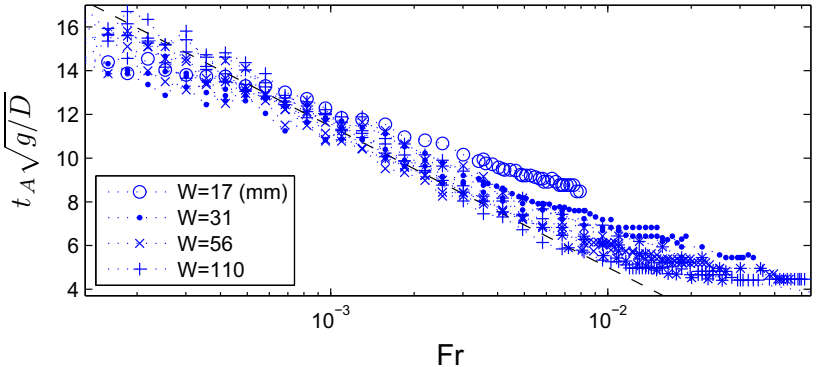

Fig. 40 Median avalanche times, scaled by $\sqrt{g / D}$, against Froude number for sand. The dashed line is the linear fit to the $(D, W)=$ $(287,110) \mathrm{mm}$ data used in Fig. 25

exploiting a fast Fourier transform or by explicitly evaluating the coefficients of its Fourier series.

The mean and standard deviation of the signal of $\theta(t)$ can be computed analytically in terms of the statistics of the random variables, after evaluating the time integrals and replacing the sum over avalanches by expectations. One finds

$$
\begin{aligned}
\langle\theta(t)\rangle= & \frac{1}{2}\left\langle\theta_{\text {start }}+\theta_{\text {stop }}\right\rangle \\
& \times\left[1+\frac{\sigma_{\text {start }}^{2}-\sigma_{\text {stop }}^{2}}{\left\langle\theta_{\text {start }}+\theta_{\text {stop }}\right\rangle\left\langle\theta_{\text {start }}-\theta_{\text {stop }}+\Omega t_{A}\right\rangle}\right]
\end{aligned}
$$

and

$\sigma^{2}=\frac{1}{12}\left\langle\theta_{\text {start }}-\theta_{\text {stop }}\right\rangle^{2}-\frac{\left(\sigma_{\text {start }}^{2}-\sigma_{\text {stop }}^{2}\right)^{2}}{4\left\langle\theta_{\text {start }}-\theta_{\text {stop }}+\Omega t_{A}\right\rangle^{2}}$ 


$$
+\frac{1}{2}\left(\sigma_{\text {start }}^{2}+\sigma_{\text {stop }}^{2}\right) \frac{\left\langle\theta_{\text {start }}-\theta_{\text {stop }}+\frac{2}{3} \Omega t_{A}\right\rangle}{\left\langle\theta_{\text {start }}-\theta_{\text {stop }}+\Omega t_{A}\right\rangle} .
$$

The model therefore rationalizes the relation between the standard deviation of the surface angle and the avalanche amplitude: $\sigma^{2} \approx \frac{1}{12}\left\langle\theta_{\text {start }}-\theta_{\text {stop }}\right\rangle^{2}+\frac{1}{2}\left(\sigma_{\text {start }}^{2}+\sigma_{\text {stop }}^{2}\right)$ when the avalanche duration is relatively small and $\sigma_{\text {start }}^{2}-\sigma_{\text {stop }}^{2} \ll$ $\langle\Delta \theta\rangle^{2}$, where $\sigma_{\text {start }}$ and $\sigma_{\text {stop }}$ are the standard deviations of the starting and stopping angles (See Figs. 29, 30, 31, 32, 33, $34,35,36,37,38,39,40)$

\section{References}

1. Bocquet, L., Charlaix, E., Ciliberto, S., Crassous, J.: Moistureinduced ageing in granular media and the kinetics of capillary condensation. Nature 396(6713), 735-737 (1998)

2. Börzsönyi, T., Halsey, T., Ecke, R.: Avalanche dynamics on a rough inclined plane. Phys. Rev. E 78(1), 011306 (2008)

3. Brucks, A., Arndt, T., Ottino, J., Lueptow, R.: Behavior of flowing granular materials under variable g. Phys. Rev. E 75(3), 032301 (2007)

4. Caponeri, M., Douady, S., Fauve, S., Laroche, C.: Dynamics of avalanches in a rotating cylinder. In: Guazzelli, E., Oger, L. (eds.) Mobile Particulate Systems, pp. 331-366. Springer, Dordrecht (1995)

5. Courrech du Pont, S., Gondret, P., Perrin, B., Rabaud, M.: Granular avalanches in fluids. Phys. Rev. Lett. 90(4), 044301 (2003)

6. Courrech du Pont, S., Gondret, P., Perrin, B., Rabaud, M.: Wall effects on granular heap stability. EPL (Europhys. Lett.) 61(4), 492 (2003)

7. Davidson, J., Scott, D., Bird, P., Herbert, O., Powell, A., Ramsay, H.: Granular motion in a rotary kiln: the transition from avalanching to rolling. KONA Powder Part. J. 18, 149-156 (2000)

8. Ding, Y., Forster, R., Seville, J., Parker, D.: Granular motion in rotating drums: bed turnover time and slumping-rolling transition. Powder Technol. 124(1), 18-27 (2002)

9. Dury, C., Ristow, G., Moss, J., Nakagawa, M.: Boundary effects on the angle of repose in rotating cylinders. Phys. Rev. E 57(4), 4491 (1998)

10. Félix, G., Falk, V., D’Ortona, U.: Granular flows in a rotating drum: the scaling law between velocity and thickness of the flow. Eur. Phys. J. E Soft Matter Biol. Phys. 22(1), 25-31 (2007)

11. Fischer, R., Gondret, P., Perrin, B., Rabaud, M.: Dynamics of dry granular avalanches. Phys. Rev. E 78(2), 021302 (2008)

12. Fischer, R., Gondret, P., Rabaud, M.: Transition by intermittency in granular matter: from discontinuous avalanches to continuous flow. Phys. Rev. Lett. 103(12), 128002 (2009)

13. Forterre, Y., Pouliquen, O.: Flows of dense granular media. Annu. Rev. Fluid Mech. 40, 1-24 (2008)

14. MiDi, G.D.R.: On dense granular flows. Eur. Phys. J. E 14, 341-365 (2004)

15. Gray, J.M.N.T.: Granular flow in partially filled slowly rotating drums. J. Fluid Mech. 441, 1-29 (2001)
16. Henein, H., Brimacombe, J., Watkinson, A.: Experimental study of transverse bed motion in rotary kilns. Metall. Trans. B 14(2), 191-205 (1983)

17. Henein, H., Brimacombe, J., Watkinson, A.: Modelling of transverse solids motion in rotary kilns. Metall. Trans. B 14(2), 207-220 (1983)

18. Jenkins, J.T., Berzi, D.: Dense inclined flows of inelastic spheres: tests of an extension of kinetic theory. Granul. Matter 12(2), 151$158(2010)$

19. Jop, P., Forterre, Y., Pouliquen, O.: Crucial role of sidewalls in granular surface flows: consequences for the rheology. J. Fluid Mech. 541, 167-192 (2005)

20. Khakhar, D., McCarthy, J., Ottino, J.: Radial segregation of granular mixtures in rotating cylinders. Phys. Fluids 9(12), 3600-3614 (1997)

21. Kleinhans, M., Markies, H., De Vet, S., Postema, F., et al.: Static and dynamic angles of repose in loose granular materials under reduced gravity. J. Geophys. Res. Planets 116(E11), 1-13 (2011)

22. Lemieux, P.A., Durian, D.: From avalanches to fluid flow: a continuous picture of grain dynamics down a heap. Phys. Rev. Lett. $\mathbf{8 5}(20), 4273$ (2000)

23. Lim, S.Y., Davidson, J., Forster, R., Parker, D., Scott, D., Seville, J.: Avalanching of granular material in a horizontal slowly rotating cylinder: Pept studies. Powder Technol. 138(1), 25-30 (2003)

24. Liu, X., Specht, E., Mellmann, J.: Slumping-rolling transition of granular solids in rotary kilns. Chem. Eng. Sci. 60(13), 3629-3636 (2005)

25. Lumay, G., Vandewalle, N.: Flow of magnetized grains in a rotating drum. Phys. Rev. E 82(4), 040301 (2010)

26. Maneval, J., Hill, K., Smith, B., Caprihan, A., Fukushima, E.: Effects of end wall friction in rotating cylinder granular flow experiments. Granul. Matter 7(4), 199-202 (2005)

27. Mellmann, J.: The transverse motion of solids in rotating cylindersforms of motion and transition behavior. Powder Technol. 118(3), 251-270 (2001)

28. Nasuno, S., Kudrolli, A., Bak, A., Gollub, J.P.: Time-resolved studies of stick-slip friction in sheared granular layers. Phys. Rev. E 58(2), 2161 (1998)

29. Orpe, A., Khakhar, D.: Scaling relations for granular flow in quasitwo-dimensional rotating cylinders. Phys. Rev. E 64(3), 031302 (2001)

30. Orpe, A., Khakhar, D.: Rheology of surface granular flows. J. Fluid Mech. 571, 1-32 (2007)

31. Pouliquen, O., Forterre, Y.: Friction law for dense granular flows: application to the motion of a mass down a rough inclined plane. J. Fluid Mech. 453, 133-151 (2002)

32. Rajchenbach, J.: Flow in powders: from discrete avalanches to continuous regime. Phys. Rev. Lett. 65(18), 2221 (1990)

33. Rajchenbach, J.: Granular flows. Adv. Phys. 49(2), 229-256 (2000)

34. Rajchenbach, J.: Dynamics of grain avalanches. Phys. Rev. Lett. 88(1), 014301 (2002)

35. Sauret, A., Balmforth, N., Caulfield, C., McElwaine, J.: Bulldozing of granular material. J. Fluid Mech. 748, 143-174 (2014)

36. Tegzes, P., Vicsek, T., Schiffer, P.: Development of correlations in the dynamics of wet granular avalanches. Phys. Rev. E 67(5), 051303 (2003) 